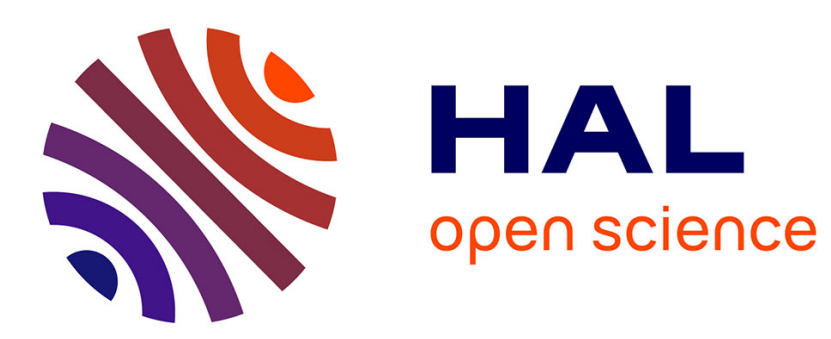

\title{
Semiclassical Asymptotics Beyond All Orders for Simple Scattering Systems
}

Alain Joye, Charles-Edouard Pfister

\section{To cite this version:}

Alain Joye, Charles-Edouard Pfister. Semiclassical Asymptotics Beyond All Orders for Simple Scattering Systems. SIAM Journal on Mathematical Analysis, 1995, 26 (4), 10.1137/S0036141093250852 . hal-01232499

\section{HAL Id: hal-01232499 \\ https://hal.science/hal-01232499}

Submitted on 24 Nov 2015

HAL is a multi-disciplinary open access archive for the deposit and dissemination of scientific research documents, whether they are published or not. The documents may come from teaching and research institutions in France or abroad, or from public or private research centers.
L'archive ouverte pluridisciplinaire $\mathbf{H A L}$, est destinée au dépôt et à la diffusion de documents scientifiques de niveau recherche, publiés ou non, émanant des établissements d'enseignement et de recherche français ou étrangers, des laboratoires publics ou privés. 


\section{SEMICLASSICAL ASYMPTOTICS BEYOND ALL ORDERS FOR} SIMPLE SCATTERING SYSTEMS *

\section{ALAIN JOYE ${ }^{\dagger}$ AND CHARLES-EDOUARD PFISTER}

Abstract. The semiclassical limit $\varepsilon \rightarrow 0$ of the scattering matrix $S$ associated with the equation $i \varepsilon \frac{d \varphi(t)}{d t}=A(t) \varphi(t)$ is considered. If $A(x)$ is an analytic $n \times n$ matrix whose eigenvalues are real and nondegenerate for all $x \in \mathbf{R}$, the matrix $S$ is computed asymptotically up to errors $O\left(e^{-\kappa \varepsilon^{-1}}\right), \kappa>$ 0 . Moreover, for the case $n=2$ and under further assumptions on the behavior of the analytic continuations of the eigenvalues of $A(x)$, the exponentially small off-diagonal elements of $S$ are given by an asymptotic expression accurate up to relative errors $O\left(e^{-\kappa \varepsilon}\right)$. The adiabatic transition probability for the time-dependent Schrodinger equation, the semiclassical above barrier reflection coeffcient for the stationary Schrodinger equation, and the total variation of the adiabatic invariant a time-dependent classical oscillator are computed asymptotically to illustrate results.

Key words. singular perturbations, turning point theory, semiclassical, and adiabatic approximation, asymptotics of $S$-matrix

AMS subject classifications. $34 \mathrm{E} 20,34 \mathrm{~L} 25,81 \mathrm{Q} 20$

1. Introduction. Let us consider the following well-known equations. The first one is the time-dependent Schrödinger equation for a two-level system

$$
i \hbar \frac{d \psi(t)}{d t}=H(\varepsilon t) \psi(t)
$$

$t \in \mathbf{R}, \psi(t) \in \mathcal{H}=\mathbf{C}^{2}$ and $H(\varepsilon t)$ is a $2 \times 2$ self-adjoint linear operator with two distinct real eigenvalues. The parameter $\varepsilon$ is positive and small. The second equation is the stationary one-dimensional Schrödinger equation

$$
-\hbar^{2} \frac{d^{2} \psi(x)}{d x^{2}}+V(x) \psi(x)=E \psi(x)
$$

$x \in \mathbf{R}, \psi(x) \in \mathbf{C}$ and $V(x)$ is a bounded real-valued function. The real parameter $E$ is chosen in such a way that

$$
E>\sup _{x \in \mathbb{R}} V(x) \text {. }
$$

The third equation is the equation of motion of a classical oscillator whose frequency varies with time

$$
\ddot{v}(t)=-\omega^{2}(\varepsilon t) v(t), \quad v(0)=u_{0}, \quad \dot{v}(0)=u_{1} .
$$

This equation is of the same type as (1.2) since we assume that the real-valued function $\omega(t)$ is bounded and such that

$$
\inf _{t \in \mathbf{R}} \omega^{2}(t)>0
$$

Received by the editors May 23,1993; accepted for publication (in revised form) January 14 1994.

Centre de Physique Théorique, Centre National de la Recherche Scientifique Marseille, Luminy Case 907, F-13288 Marseille Ceder 9, France.

‡Département de Mathématiques, Ecole Polytechnique Fédérale de Lausanne, CH-1015 Lausanne, Switzerland. 
For the first two equations we are interested in the behavior of the solution for $t \rightarrow+\infty$ or $x \rightarrow+\infty$, when the behavior for $t \rightarrow-\infty$ or $x \rightarrow-\infty$ is fixed. Moreover we want to analyze this scattering situation when $\varepsilon$ tends to zero and $\hbar=1$ for equation (1.1), the so-called adiabatic limit, or $\hbar$ tends to zero for equation (1.2), the so-called semiclassical limit. For the initial value problem (1.4), we consider the adiabatic invariant $J$ defined as twice the ratio of the energy to the frequency

$$
J(t, \varepsilon)=\frac{|\dot{v}(t)|^{2}+\omega^{2}(\varepsilon t)|v(t)|^{2}}{\omega(\varepsilon t)}
$$

in the limit $\varepsilon \rightarrow 0$. More precisely, we are interested in its total variation during the whole evolution

$$
\Delta J(\varepsilon) \equiv J(+\infty, \varepsilon)-J(-\infty, \varepsilon) .
$$

In this respect, we consider (1.4) more as a scattering problem than as an initial value problem. All three problems are very closely related. Let $x=\varepsilon t$ be a rescaled time for equations (1.1) and (1.4). Then equation (1.1) becomes with $\varphi(x)=\psi(t(x))$ and $\hbar=1$

$$
i \varepsilon \frac{d \varphi(x)}{d x}=H(x) \varphi(x) .
$$

On the other hand, defining $u(x)=v(t(x))$ and

$$
\varphi(x)=\left(\begin{array}{c}
u(x) \\
i \varepsilon \frac{d u(x)}{d x}
\end{array}\right)
$$

equation (1.4) is equivalent to

$$
i \varepsilon \frac{d \varphi(x)}{d x}=\left(\begin{array}{cc}
0 & 1 \\
\omega^{2}(x) & 0
\end{array}\right) \varphi(x), \quad \varphi(0)=\left(\begin{array}{c}
u_{0} \\
i u_{1}
\end{array}\right) .
$$

Similarly, with

$$
\varphi(x)=\left(\begin{array}{c}
\psi(x) \\
i \varepsilon \frac{d \psi(x)}{d x}
\end{array}\right)
$$

and setting $\hbar=\varepsilon$, equation.(1.2) becomes

$$
i \varepsilon \frac{d \varphi(x)}{d x}=\left(\begin{array}{cc}
0 & 1 \\
E-V(x) & 0
\end{array}\right) \varphi(x) .
$$

Thus the three equations (1.7), (1.9), and (1.11) are particular cases of

$$
i \varepsilon \frac{d \varphi(x)}{d x}=A(x) \varphi(x)
$$

where $A(x)$ is a linear operator on $\mathcal{H}=\mathbf{C}^{2}$ with two distinct real eigenvalues. Our purpose is to study a scattering problem for (1.12) in the "semiclassical" limit $\varepsilon$ tends to zero under the hypothesis that $A(x)$ is analytic, has two distinct real eigenvalues for all $x \in \mathbf{R}$, and has well-defined limits when $x \rightarrow \pm \infty$. It is natural to express the solutions of (1.12) as linear combinations of eigenvectors of $A(x)$ :

$$
\varphi(x)=\sum_{i=1}^{2} c_{j}(x) e^{-i / \varepsilon \int_{0}^{x} e_{j}\left(x^{\prime}\right) d x^{\prime}} \varphi_{j}(x)
$$

where $A(x) \varphi_{j}(x)=e_{j}(x) \varphi_{j}(x)$. Our conditions on the behavior of $A(x)$ for large $|x|$ imply that

$$
\lim _{x \rightarrow \pm \infty} c_{j}(x)=c_{j}( \pm \infty)
$$

exist, so that the following scattering problem is well defined:

Given $c_{j}(-\infty), j=1,2$ find $c_{j}(+\infty), j=1,2$, i.e., find the matrix $S$ defined by

$$
\left(\begin{array}{l}
c_{1}(+\infty) \\
c_{2}(+\infty)
\end{array}\right)=\left(\begin{array}{ll}
S_{11} & S_{12} \\
S_{21} & S_{22}
\end{array}\right)\left(\begin{array}{l}
c_{1}(-\infty) \\
c_{2}(+\infty)
\end{array}\right)
$$

There is a "canonical" choice of eigenvectors of $A(x)$ specified (up to a global factor) by the condition

$$
P_{j}(x) \frac{d \varphi_{j}(x)}{d x}=0
$$

where $P_{j}(x)$ is the eigenprojection corresponding to $e_{j}(x)$. Condition (1.16) has a geometrical interpretation in terms of parallel transport which we give below. In particular, it is immediate to verify that for $A(x)$ given by (1.9) or by (1.11) with the identification $\omega^{2}(x) \equiv E-V(x)$, the eigenvectors associated with $e_{j}(x)=(-1)^{j} \omega(x)$,

$$
\varphi_{1}(x)=\left(\begin{array}{c}
\frac{1}{\sqrt{\omega(x)}} \\
-\sqrt{\omega(x)}
\end{array}\right), \quad \varphi_{2}(x)=\left(\begin{array}{c}
\frac{1}{\sqrt{\omega(x)}} \\
+\sqrt{\omega(x)}
\end{array}\right)
$$

satisfy (1.16), so that (1.13) gives the solutions of (1.9) and (1.11) as superpositions of the two well-known Wentzel-Kramers-Brillouin (WKB) functions

$$
e^{-i / \varepsilon \int_{0}^{x} e_{j}\left(x^{\prime}\right) d x^{\prime}} \varphi_{j}(x) .
$$

When this choice of eigenvectors is made, a solution $\varphi(x)$ of (1.12) characterized by $c_{j}(-\infty)=1$ and $c_{k}(-\infty)=0, k \neq j$, satisfies

$$
\sup _{x \in \mathbb{R}}\left|\varphi(x)-e^{-i / \varepsilon \int_{0}^{x} e_{j}\left(x^{\prime}\right) d x^{\prime}} \varphi_{j}(x)\right|=O(\varepsilon)
$$

Consequently,

$$
S=1+O(\varepsilon)
$$

The approximations (1.19) and (1.20) are true without assuming analyticity of $A(x)$. On the other hand, if analyticity holds, we can approximate the solutions of (1.12) and 
thus determine the matrix $S$ up to error terms $O\left(\exp \left(-\kappa \varepsilon^{-1}\right)\right), \kappa>0$ (see Corollary $2.5)$

$$
S_{k j}=s_{j}(\varepsilon) \delta_{k j}+O\left(\exp \left(-\kappa \varepsilon^{-1}\right)\right),
$$

where $\left|s_{j}(\varepsilon)\right|=O(1)$. These results are corollaries of the iterative scheme presented in $\S 2$, which will be used in $\S 3$. Actually they are derived for $A(x)$ a $n \times n$ matrix whose eigenvalues are assumed to be real and nondegenerate for any $x \in \mathbf{R}$.

The asymptotic formulae (1.21) imply in particular that the nondiagonal terms of $S$ are $O\left(\exp \left(-\kappa \varepsilon^{-1}\right)\right)$. These terms are important in applications because they are related, for equation (1.1), to the probability of a quantum transition between the two levels of the system or, in the case of equation (1.2), to the above barrier reflection coefficient and, in the case of equation (1.4) to the quantity $\Delta J(\varepsilon)$. Under furthe hypotheses on the analytic behavior of the eigenvalues of $A(x)$ we show that it is possible to find an asymptotic expression for $S_{21}$ or $S_{12}$ accurate up to exponentially pmall relative corections. The asymptotic formula is expressed by means of the complex degeneracy points of the analytic continuations of eigenvalues $e_{j}(x)$. If there are $p$ contributing degeneracy points, the asymptotic expression reads (see Theorem 3.7 and (2.43), (2.45))

$$
S_{21}=\sum_{k=0}^{p} e^{-i \theta^{\star}(k, \varepsilon)} e^{-i \gamma^{*}(k, \varepsilon) \varepsilon^{-1}}+e^{-\tau \varepsilon^{-1}} O\left(e^{-\kappa \varepsilon^{-1}}\right), \quad \kappa, \tau>0
$$

where $\theta^{\star}(k, \varepsilon)$ is $O(1)$ and $\operatorname{Im} \gamma^{\star}(k, \varepsilon)=-\tau+O\left(\varepsilon^{2}\right), k=1, \ldots, p$. It should be noted that the error term is smaller by an exponentially decreasing factor than the least significant term in the sum (122). This asymptotic formula is proven in $\$ 3$, which is the main part of the paper. It is obtained by combining our iterative scheme with a method due to Fröman and Fröman [1]. We give in $\S 4$ explicit formulae in terms of
$A(x)$ for the expressions $\theta^{\star}(k, \varepsilon)$ and $\gamma^{\star}(k, \varepsilon)$ appearing in (1.22). The consequences $A(x)$ for the expressions $\theta^{\star}(k, \varepsilon)$ and $\gamma^{\star}(k, \varepsilon)$ appearing in (1.22). The consequences formulated in $\$ 4$ as well. Finally, we give in the appendix an explicit example which is shown numerically to fit in the framework developed in this paper.

Let us come back to the choice of eigenvectors satisfying (1.16). Let $M$ be some manifold, which we suppose to be embedded in $\mathbf{R}^{q}$, and let $P$ be a smooth projectionvalued map, $m \mapsto P(m)$, defined on $M, P(m)$ being a projection (not necessarily orthogonal) of some given Hilbert space. The map $P$ defines a bundle $F$ with base $M$, whose fiber over $m^{\prime} \in M$ is the set of elements $\left(m^{\prime}, \phi\right)$ with $\phi \in P\left(m^{\prime}\right) \mathcal{H}$. The bundle $F$ is embedded in the trivial bundle $\mathbf{R}^{q} \times \mathcal{H}$ and has a natural connection defined by $P$. Ind $f(t)$ vector of a curve $c(t)=\left(c_{1}(t), c_{2}(t)\right)$ with $c_{2}(t)=P\left(c_{1}(t)\right) c_{2}(t)$ and $c(0)=f$, i.e., $v_{f}=\left(\dot{c}_{1}(0), \dot{c}_{2}(0)\right)_{f}$. The vertical vectors at $f$ are velocity vectors of curves $c(t)$ with $c_{1}(t) \equiv m$; since in this case $c_{2}(t) \in P(m) \mathcal{H}$ for all $t$, they are of the form $\left(0, \dot{c}_{2}(0)\right)_{f}$ with $\dot{c}_{2}(0) \in P(m) \mathcal{H}$. Conversely, since $c_{2}(t)=P\left(c_{1}(t)\right) c_{2}(t)$, any vector of the form $\left(0, \dot{c}_{2}(0)\right)_{f}$ is vertical. Therefore, we have a decomposition of $v_{f}$ into a vertical vector $\left(0, P(m) \dot{c}_{2}(0)\right)$ and a horizontal vector $\left(\dot{c}_{1}(0),(1-P(m)) \dot{c}_{2}(0)\right\rangle_{f}$, hence a connection $\left(0, P(m) c_{2}(0)\right) f$ and a horizon and $\phi(t) \in P(\gamma(t)) \mathcal{H}$ be a vector field is parallel if and only if the velocity vector $(\dot{\gamma}(t), \dot{\phi}(t))_{\gamma(t)}$ is horizontal for all $t$, i.e., if and only if $P(\gamma(t)) \dot{\phi}(t)=0$, which is precisely (1.16).

Before ending this introduction let us make some very brief comments on the vast amount of literature devoted to the exponential decay of nondiagonal elements of the matrix $S$. We do not attempt at all to give an exhaustive account of it, but we want to set our work in context relative to the main results. We quote these results according to their content and not chronologically. The reader may find further references in the books [2] and [3]. The intermediate result (1.21) is not new, see [2], [3] and references therein, but we nevertheless obtain a new derivation of it in $\$ 2$. For recent related results see also [4]. The asymptotic expression (1.22) generalizes several rigorous results which were obtained either in the case of equations (1.7) and (1.11) or in the study of $\Delta J(\varepsilon)$. When one complex eigenvalue degeneracy only contributes, it has been known since publication of the works [1], [5], [6] that

$$
S_{21}=e^{-i \theta} e^{-i \gamma \varepsilon^{-1}}+O(\varepsilon) e^{\operatorname{Im} \gamma \varepsilon^{-1}}, \quad \operatorname{Im} \gamma<0
$$

with $\theta=\pi / 2$ for equation (1.11) and, providing $A(x)$ is a real symmetric matrix, for equation (1.7) as well. It was shown recently that when $A(x)$ is a hermitian matrix in (1.7), $\theta$ can take any complex value [7], see also [8]. A corresponding asymptotic expression for $\Delta J(\varepsilon)$ in this situation can be found in [9]-[11]. See also [12] for more recent related results. The expression (1.23) was then generalized in two ways for equations (1.7) and (1.11). First, when several eigenvalue degeneracy points contribute to the asymptotics of $S_{21}$, it was proven using standard stretching and matching techniques that [5], [13]

$$
S_{21}=\sum_{k=0}^{p} e^{-i \theta(k)} e^{-i \gamma(k) \varepsilon^{-1}}+O\left(\varepsilon^{\alpha}\right) e^{\operatorname{Im} \gamma \varepsilon^{-1}},
$$

where $0<\alpha<1$ and $\operatorname{Im} \gamma(k)=\operatorname{Im} \gamma<0 \forall k$. The leading term of (1.24) gives rise to the so called "Stückelberg oscillations" as $\varepsilon \rightarrow 0$, a phenomenon which is illustrated numerically in [13]. Note also that the error term is $O\left(\varepsilon^{\alpha}\right)$ instead of $O(\varepsilon)$, which is a common drawback of the method employed to get (1.24). Then, higher-order corrections to formula (1.23) were studied systematically in [14], [15] for equation (1.11) and in [16] for equation (1.7):

$$
S_{21}=e^{-i \theta^{q}(\varepsilon)} e^{-i \gamma^{q}(\varepsilon) \varepsilon^{-1}}+O\left(\varepsilon^{q+1}\right) e^{-\tau \varepsilon^{-1}} \quad \forall q \in \mathrm{N}, \tau>0,
$$

where $\operatorname{Im} \gamma^{q}(\varepsilon)=-\tau+O\left(\varepsilon^{2}\right)$ and $\theta^{q}(\varepsilon)=O(1)$. The iterative scheme of $\S 2$ was introduced in [16] to derive this expression in the adiabatic context. Thus the asymptotic expression (1.22) captures all the features of these previous results and it holds for more general situations than those described by the particular matrices in (1.7) and (1.11). Moreover, it yields an expression accurate up to exponentially small corrections for the logarithm of $S_{21}$ since we can write for $p=1$

$$
\ln S_{21}=-i \frac{\gamma^{\star}(\varepsilon)}{\varepsilon}-i \theta^{\star}(\varepsilon)+O\left(e^{-\kappa \varepsilon^{-1}}\right) .
$$

2. Approximate solution. The results of this section will be used in $\S 3$. We consider a slightly more general problem than in the introduction. Let $\mathcal{H}=\mathrm{C}^{n}$, with the usual scalar product, and $A(x), x \in \mathbf{R}$, be a linear operator on $\mathcal{H}$. We study the equation $\left({ }^{\prime}=\frac{d}{d x}\right)$

$$
i \varepsilon U^{\prime}\left(x, x_{0}\right)=A(x) U\left(x, x_{0}\right)
$$

$$
U\left(x_{0}, x_{0}\right)=1
$$


under the condition that $A(x)$ is analytic in $x$ and for each $x$ the spectrum of $A(x)$ consists of $n$ distinct real eigenvalues $e_{1}(x)<\cdots<e_{n}(x)$, with corresponding eigenprojections $P_{1}(x), \ldots, P_{n}(x)$. Note that the evolution $U$ is not unitary in general.

In order to find an approximate solution of (2.1) we first consider another problem. Let $\psi(x)$ be a solution of

$$
i \varepsilon \psi^{\prime}(x)=A(x) \psi(x)
$$

If $Q\left(x_{0}\right)$ is a projection such that $Q\left(x_{0}\right) \psi\left(x_{0}\right)=\psi\left(x_{0}\right)$, then for any $x$ we have a projection $Q(x)$ such that $Q(x) \psi(x)=\psi(x)$. Indeed, if $U\left(x, x_{0}\right)$ is the solution of (2.1) such that $U\left(x_{0}, x_{0}\right)=1$, we take

$$
Q(x)=U\left(x, x_{0}\right) Q\left(x_{0}\right) U\left(x_{0}, x\right)
$$

The projection $Q(x)$ is a solution of

$$
i \varepsilon Q^{\prime}(x)=[A(x), Q(x)]
$$

with the notation $[A, B] \equiv A B-B A$. Let us suppose that at $x_{0}$ we have a complete set of projections $Q_{j}\left(x_{0}\right)$, i.e., $Q_{j}\left(x_{0}\right) Q_{k}\left(x_{0}\right)=Q_{k}\left(x_{0}\right) \delta_{j k}, \sum_{j} Q_{j}\left(x_{0}\right)=1$. Then the $Q_{j}(x)$ form a complete set of projections as well and using the fact that for any projection $P(x)$ we have $P(x) P^{\prime}(x) P(x)=0$, it follows that

$$
Q_{j}^{\prime}(x)=\left[\sum_{m} Q_{m}^{\prime}(x) Q_{m}(x), Q_{j}(x)\right] .
$$

Therefore we have for all $j$

$$
\left[A(x)-i \varepsilon \sum_{m} Q_{m}^{\prime}(x) Q_{m}(x), Q_{j}(x)\right]=0
$$

We look for approximate solutions of this equation. Since $\left[A(x), P_{j}(x)\right] \equiv 0$, the eigenprojections $P_{j}(x)$ are approximate solutions of (2.6) up to an error term $O(\varepsilon)$ Let

$$
A_{1}(x):=A(x)-i \varepsilon K_{0}(x)
$$

with

$$
K_{0}(x):=\sum_{m} P_{m}^{\prime}(x) P_{m}(x)
$$

By perturbation theory, if $\varepsilon$ is small enough, $A_{1}(x)$ has $n$ distinct eigenvalues $e_{1, j}(x)$ with corresponding eigenprojections $P_{1, j}(x), j=1, \ldots, n$, such that $e_{1, j}(x)=e_{j}(x)+$ $O\left(\varepsilon^{2}\right)$, and $P_{1, j}(x)=P_{j}(x)+O(\varepsilon)$. Indeed, $e_{1, j}(x)=e_{j}(x)-i \varepsilon \operatorname{tr}\left(P_{j}(x) K_{0}(x)\right)+$ $O\left(\varepsilon^{2}\right)$ and $P_{j}(x) K_{0}(x) P_{j}(x)=0$. The projections $P_{1, j}(x)$ are approximate solutions of (2.6) up to an error term $O\left(\varepsilon^{2}\right)$ since $\left[A_{1}(x), P_{1, j}(x)\right]=0$. Let

$$
K_{1}(x):=\sum_{m} P_{1, m}^{\prime}(x) P_{1, m}(x)
$$

$$
A_{2}(x):=A(x)-i \varepsilon K_{1}(x) .
$$

Again, for $\varepsilon$ small enough, $A_{2}(x)$ has $n$ distinct eigenvalues $e_{2, j}(x)$ with corresponding eigenprojections $P_{2, j}(x)$. Since $A_{2}(x)=A_{1}(x)+i \varepsilon\left(K_{0}(x)-K_{1}(x)\right)$ and $K_{0}(x)-$ $K_{1}(x)=O(\varepsilon), P_{2 j}(x)$ is an approximate solution of $(2.6)$ up to an error term $O\left(\varepsilon^{3}\right)$ We can iterate this procedure. At the $q$ th iteration we have approximate solutions $P_{q, j}(x)$, up to order term $O\left(\varepsilon^{q+1}\right)$, which are eigenprojections of

$$
A_{q}(x):=A(x)-i \varepsilon K_{q-1}(x)
$$

with

$$
K_{q-1}(x)=\sum_{m} P_{q-1, m}^{\prime}(x) P_{q-1, m}(x)
$$

We now construct approximate solutions for (2.1). Let $Q_{m}(x)$ be a complete smooth family of projections of $\mathcal{H}, Q_{m}(x) Q_{n}(x)=\delta_{m n} Q_{m}(x)$ and $\sum_{m} Q_{m}(x)=1$. We say that an evolution $V\left(x, x^{\prime}\right),\left(V\left(x^{\prime}, x^{\prime}\right)=1, V\left(x_{2}, x_{1}\right) V\left(x_{1}, x_{0}\right)=V\left(x_{2}, x_{0}\right)\right)$, follows the decomposition of $\mathcal{H}$,

$$
\mathcal{H}=\bigoplus_{m} Q_{m}(x) \mathcal{H}
$$

if for all $x, x^{\prime}$

$$
Q_{m}(x) V\left(x, x^{\prime}\right)=V\left(x, x^{\prime}\right) Q_{m}\left(x^{\prime}\right) .
$$

It is known (see [17] or [18]) that a smooth evolution with property (2.13) is the solution of an equation of the type

(2.14) $\quad V^{\prime}\left(x, x_{0}\right)=\left(B(x)+\sum_{m} Q_{m}^{\prime}(x) Q_{m}(x)\right) V\left(x, x_{0}\right), \quad V\left(x_{0}, x_{0}\right)=\mathbf{1}$

where $B(x)$ is such that

$$
\left[B(x), Q_{m}(x)\right]=0 \quad \forall m
$$

Reciprocally, any smooth evolution satisfying (2.14) and (2.15) possesses the intertwining property (2.13). The idea is to construct approximate solutions of $(2.1)$ by choosing evolutions which follow the decomposition of $\mathcal{H}$ into

$$
\mathcal{H}=\bigoplus_{m} P_{q, m}(x) \mathcal{H}
$$

Therefore we define $U_{q}\left(x, x_{0}\right)$ as the solution of

(2.17) $\quad i \varepsilon U_{q}^{\prime}\left(x, x_{0}\right)=\left(A_{q}(x)+i \varepsilon K_{q}(x)\right) U_{q}\left(x, x_{0}\right), \quad U_{q}\left(x_{0}, x_{0}\right)=1$

The next lemma, which is actually Proposition 2.1 of [19], gives the main estimate which we need to control the error term for the approximate solution $U_{q}\left(x, x_{0}\right)$. This lemma is also used in $\S 3$. 
For any $z \in \mathbf{C}$ and $r>0$ let $D(z ; r)=\left\{z^{\prime} \in \mathbf{C}:\left|z^{\prime}-z\right|<r\right\}$ and $\partial D(z ; r)=\left\{z^{\prime} \in\right.$ $\left.\mathbf{C}:\left|z^{\prime}-z\right|=r\right\}$. Given $z_{0} \in \mathbf{C}$ and $r_{0}>0$ let $A(z)$ be analytic in $D\left(z_{0} ; r_{0}\right)$ with a spectrum consisting of $n$ distinct eigenvalues $e_{j}(z)$ with corresponding eigenprojection $P_{j}(z)$ for all $z \in D\left(z_{0} ; r_{0}\right)$. We define $A_{q}(z), K_{q}(z), P_{q, j}(z)$, and $e_{q, j}(z)$ as above by the iteration method based on (211) and (212). We set $R(z, \lambda)=(A(z)-\lambda 1)^{-1}$.

LEMMA 2.1. Let $z_{0} \in \mathbf{C}, r_{0}>0$ and $A(z)$ be defined on $D\left(z_{0} ; r_{0}\right)$ with the above properties. Let $r_{1}>0$ and $D_{j}:=D\left(e_{j}\left(z_{0}\right) ; 2 r_{1}\right)$ be $n$ disjoint discs in $\mathbf{C}, j=1, \ldots, n$, such that for all $z \in D\left(z_{0} ; r_{0}\right)$

$$
e_{j}(z) \in D\left(e_{j}\left(z_{0}\right) ; r_{1}\right)
$$

Let

$$
a=a\left(z_{0}\right):=\sup _{j} \sup _{\lambda \in \partial D_{j}} \sup _{z \in D\left(z_{0} ; r_{0}\right)}\|R(z, \lambda)\|<\infty
$$

and

$$
b=b\left(z_{0}\right):=\sup _{z \in D\left(z_{0} ; r_{0}\right)}\left\|K_{0}(z)\right\|<\infty .
$$

Then there exist $\varepsilon^{\star}=\varepsilon^{\star}(a, b)>0$ and $c=c\left(r_{0}, r_{1}, a, b\right)<\infty$ such that

$$
\left\|K_{q}(z)-K_{q-1}(z)\right\| \leq b \varepsilon^{q} c^{q} q !
$$

and

\section{$\left\|K_{q}(z)\right\| \leq 2 b$}

for all $z \in D\left(z_{0} ; r_{0}\right)$, all $0<\varepsilon \leq \varepsilon^{\star}$, and all $q \leq q^{\star}(\varepsilon)=\left[\frac{1}{\text { ecE }}\right]$, where $[y]$ is the integer part of $y$ and $e$ is the basis of the neperian logarithm.

Remark. The proof of this lemma is given in [19] for the case $P_{1}+P_{2}=1$ in the general situation where the spectrum of the (possibly unbounded) operator $A(z)$ is separated in two parts for any $z \in D\left(z_{0}, r_{0}\right)$ and $\operatorname{dim} P_{1}(z) \mathcal{H}<\infty$. However, the proof is the same for the case $\sum_{j=1}^{n} P_{j}=1, n \geq 2$, apart from the obvious changes due to is the same for the case $\sum_{j=1}^{n} P_{j}=1, n \geq 2$
the presence of more than two projectors.

COROLLARY 2.2. Let the hypothesis of Lemma 2.1 be satisfied. Then for all $q \leq q^{\star}$

$$
e_{q, j}(z)=e_{j}(z)+O\left(b \varepsilon^{2}\right) \text {. }
$$
have

Proof. Since $P_{j}(z) K_{0}(z) P_{j}(z)=0$ the statement is true for $q=1$. For $q \geq 2$ we

(2.18) $\left\|A_{q}(z)-A_{1}(z)\right\| \leq \varepsilon \sum_{m=1}^{q-1}\left\|K_{m}(z)-K_{m-1}(z)\right\| \leq \varepsilon b \sum_{m=1}^{q^{*}} \varepsilon^{m} c^{m} m !=O\left(\varepsilon^{2} b\right)$

and therefore the statement follows from perturbation theory.

We now apply Lemma 2.1 and Corollary 2.2 to control the norm of $U_{q}\left(x, x_{0}\right)$. It is crucial that $U_{q}$ follows the decomposition of $\mathcal{H}$ into $\bigoplus_{m \geq 1} P_{q, m}(z) \mathcal{H}$.

CorollarY 2.3. Let $r_{0}>0$ be such that for each $x \in \mathbf{R}$ the hypotheses of Lemma 2.1 are satisfied on $D\left(x ; r_{0}\right)$ with constants $r_{1}$ and $a$ independent of $x$ and with constants $b(x) \leq b<\infty$. Then for $\varepsilon \leq \varepsilon^{\star}$ and $q \leq q^{\star}$

$$
\left\|U_{q}\left(x ; x_{0}\right)\right\| \leq \exp \left\{O\left(\left|\int_{x_{0}}^{x} b\left(x^{\prime}\right) d x^{\prime}\right|\right)\right\} .
$$

Proof. We introduce the evolution $W_{q}\left(x, x_{0}\right)$,

$$
W_{q}^{\prime}\left(x, x_{0}\right)=K_{q}(x) W_{q}\left(x, x_{0}\right), \quad W_{q}\left(x_{0}, x_{0}\right)=\mathbf{1} .
$$

From Lemma 2.1 we have

$$
\left\|W_{q}\left(x, x_{0}\right)\right\| \leq \exp \left(2\left|\int_{x_{0}}^{x} b\left(x^{\prime}\right) d x^{\prime}\right|\right)
$$

Let us choose $n$ eigenvectors $\varphi_{q, j}(0)$ of $A_{q}(0)$ at $x=0$. The vectors

$$
\varphi_{q, j}(x):=W_{q}(x, 0) \varphi_{q, j}(0), \quad j=1, \ldots, n
$$

are eigenvectors of $A_{q}(x)$ since $W_{q}(x, 0)$ interpolates between $P_{q, m}(0)$ and $P_{q, m}(x) \forall m \leq n$ (see (2.13) and (2.14)) and by definition

$$
P_{q, j}(x) \varphi_{q, j}^{\prime}(x)=0, \quad j=1, \ldots, n .
$$

Let us write $U_{q}\left(x, x_{0}\right):=W_{q}\left(x, x_{0}\right) \Phi_{q}\left(x, x_{0}\right)$. The unknown operator $\Phi_{q}\left(x, x_{0}\right)$ is the solution of

$$
\begin{aligned}
i \varepsilon \Phi_{q}^{\prime}\left(x, x_{0}\right) & =W_{q}\left(x_{0}, x\right) A_{q}(x) W_{q}\left(x, x_{0}\right) \Phi_{q}\left(x, x_{0}\right), \\
\Phi_{q}\left(x_{0}, x_{0}\right) & =1
\end{aligned}
$$

The operator $W_{q}\left(x_{0}, x\right) A_{q}(x) W_{q}\left(x, x_{0}\right)$ has eigenvalues $e_{q, j}(x)$ with eigenvectors $\varphi_{q, j}\left(x_{0}\right)$. Therefore

(2.24) $\Phi_{q}\left(x, x_{0}\right) \varphi_{q, j}\left(x_{0}\right)=\exp \left(-i \varepsilon^{-1} \int_{x_{0}}^{x} e_{q, j}\left(x^{\prime}\right) d x^{\prime}\right) \varphi_{q, j}\left(x_{0}\right), \quad j=1, \ldots, n$.

From Corollary 2.2 and the reality of $e_{j}(z)$,

hence

$$
\left|\operatorname{Im}\left(\int_{x_{0}}^{x} e_{q, j}\left(x^{\prime}\right) d x^{\prime}\right)\right| \leq O\left(\varepsilon^{2}\right)\left|\int_{x_{0}}^{x} b\left(x^{\prime}\right) d x^{\prime}\right|
$$

$$
\left\|U_{q}\left(x, x_{0}\right)\right\| \leq \exp \left\{(2+O(\varepsilon))\left|\int_{x_{0}}^{x} b\left(x^{\prime}\right) d x^{\prime}\right|\right\}
$$

Note that in the above proof we have factorized the evolution $U_{q}\left(x, x_{0}\right)$ as the product

$$
U_{q}\left(x, x_{0}\right)=W_{q}\left(x, x_{0}\right) \Phi_{q}\left(x, x_{0}\right),
$$

where $\Phi_{q}$ only is singular in the limit $\varepsilon \rightarrow 0$ and $\left\|\Phi_{q}\right\|=O(1),\left\|W_{q}\right\|=O(1)$. Since in our simple case $\Phi_{q}$ is known explicitly, the solation $\psi(x)$ of

$$
\begin{aligned}
i \varepsilon \psi^{\prime}(x) & =\left(A_{q}(x)+i \varepsilon K_{q}(x)\right) \psi(x) \\
\psi\left(x_{0}\right) & =\psi_{0}
\end{aligned}
$$


can be written as

$$
\begin{aligned}
\psi(x) & =U_{q}\left(x, x_{0}\right) \psi(0) \\
& =\sum_{j \geq 1} c_{q, j}\left(x_{0}\right) \exp \left(-i \varepsilon^{-1} \int_{x_{0}}^{x} e_{q, j}\left(x^{\prime}\right) d x^{\prime}\right) \dot{\varphi}_{q, j}(x),
\end{aligned}
$$

$$
\text { fined by the identity }
$$

where the $c_{q, j}\left(x_{0}\right)$ are defined by the identity

$$
\psi_{0}=\sum_{j \geq 1} c_{q, j}\left(x_{0}\right) \varphi_{q, j}\left(x_{0}\right)
$$

THEOREM 2.4. Let $r>0$ and $g>0$ and let $A(x)$ be analytic in $\Omega_{r}=\{z=x+i y$ : $x, y \in \mathbf{R},|y|<r\}$. Let the spectrum of $A(x)$ consist of $n$ real distinct eigenvalues $e_{j}(x), j=1, \ldots, n$, such that for all $x \in \mathbf{R}$

$$
\left|e_{k}(x)-e_{j}(x)\right| \geq g, \quad k \neq j
$$

Let

$$
\left\|K_{0}(x)\right\|=\left\|\sum_{j \geq 1} P_{j}^{\prime}(x) P_{j}(x)\right\|
$$

be an integrable function of $x$ which tends to zero as $|x| \rightarrow 0$. Then there exist constants $\varepsilon^{\star}>0, C^{\prime}<\infty, \kappa>0$ such that the above-constructed matrix $U_{q^{\star}}\left(x, x_{0}\right)$ approximates the solution $U\left(x, x_{0}\right)$ of the equation

$$
\begin{aligned}
i \varepsilon U^{\prime}\left(x, x_{0}\right) & =A(x) U\left(x, x_{0}\right), \\
U\left(x_{0}, x_{0}\right) & =1
\end{aligned}
$$

in such a way that

$$
\sup _{x, x_{0} \in \mathrm{R}}\left\|U\left(x, x_{0}\right)-U_{q^{\star}}\left(x, x_{0}\right)\right\| \leq C^{\prime} \exp \left(-\kappa \varepsilon^{-1}\right) .
$$

Remarks. i) Neither $U$ nor $U_{q^{\star}}$ are unitary in general; however, both their norms are $O(1)$ as $\varepsilon \rightarrow 0$.

ii) Note that $\lim _{x \rightarrow \pm \infty} A(x)$ need not exist, since we only require that $\lim _{x \rightarrow \pm \infty} P_{j}(x)=P_{j}( \pm \infty)$ exists.

iii) The exponential decay rate is given by $\kappa=1 / e c$ (see (2.33)) where $c$ is defined in Lemma 2.1. The decay rate obtained by this method is certainly not optimal but has the merit, however, to be explicit and rather simple to determine. It should be noted also that in the general case (i.e., $n>2$ ), it is an open problem to determine the optimal decay rate.

iv) Similar results were also obtained by different methods: Nenciu [20] considered and studied a formal series expansion in $\varepsilon$ satisfying (2.4) and Martinez [21] and Sjöstrand [22] used microlocal analysis techniques. In particular, the question raised in the preceding remark is addressed in [21]. However, the estimates needed in $\S 3$ are proved in [19] only.

Proof. By standard arguments of perturbation theory we can verify the hypothesis of Corollary 2.3 with $b(x)$ integrable on $\mathbf{R}$ (see, e.g., $\$ 2$ of [23]). We recall that

$$
q^{\star}(\varepsilon)=\left[\frac{1}{e c \varepsilon}\right]
$$

as defined in Lemma 2.1. The operator $R(x):=U_{q^{*}}\left(x_{0}, x\right) U\left(x, x_{0}\right)$ is a solution of

$$
\begin{aligned}
i \varepsilon R^{\prime}(x) & =U_{q^{*}}\left(x_{0}, x\right)\left(-A_{q^{*}}(x)-i \varepsilon K_{q^{\star}}(x)+A(x)\right) U_{q^{\star}}\left(x, x_{0}\right) R(x) \\
& =i \varepsilon U_{q^{\star}}\left(x_{0}, x\right)\left(K_{q^{*}-1}(x)-K_{q^{\star}}(x)\right) U_{q^{\star}}\left(x, x_{0}\right) R(x) .
\end{aligned}
$$

From the integrability of $b(x)$ and Lemma 2.1 we have

$$
\begin{aligned}
\|R(x)-1\| & \leq C^{\prime \prime}(c \varepsilon) q^{\star} q^{\star} ! \\
& \leq C^{\prime \prime}\left(c \varepsilon q^{\star}\right) q^{*} \\
& \leq e C^{\prime \prime} \exp \left(-\kappa \varepsilon^{-1}\right)
\end{aligned}
$$

where $\kappa=\frac{1}{\varepsilon c}$. Hence

$$
\begin{aligned}
\left\|U\left(x, x_{0}\right)-U_{q^{*}}\left(x, x_{0}\right)\right\| & \leq\left\|U_{q^{*}}\left(x, x_{0}\right)\right\|\|R(x)-\mathbf{1}\| \\
& \leq C^{\prime} \exp \left(-\kappa \varepsilon^{-1}\right) .
\end{aligned}
$$

We assume that the hypotheses of Theorem 2.4 are satisfied and we determine the matrix $S$ up to an error term $O\left(e^{-\kappa \varepsilon^{-1}}\right)$. Since $\left\|K_{0}(x)\right\|$ and thus $\left\|K_{q-1}(x)\right\|$ tend to zero at infinity in an integrable way (see Lemma 2.1 and Corollary 2.3),

$$
\lim _{x \rightarrow \pm \infty}\left\|A_{q}(x)-A(x)\right\|=0 \quad \forall q \leq q^{\star}
$$

and for all $q \leq q^{\star}$, there exist $W_{q}\left( \pm \infty, x_{0}\right)$ such that

$$
\lim _{x \rightarrow \pm \infty} W_{q}\left(x, x_{0}\right)=W_{q}\left( \pm \infty, x_{0}\right)
$$

Let us choose a point $x_{0}$ and a set of eigenvectors $\varphi_{j}\left(x_{0}\right)$ of $A\left(x_{0}\right), j=1, \ldots, n$. Using $W_{0}\left(x, x_{0}\right)$ we define a set of eigenvectors of $A(x)$ for all $x$,

$$
\varphi_{j}(x)=W_{0}\left(x, x_{0}\right) \varphi_{j}\left(x_{0}\right)
$$

Let $\psi$ be a solution of

$$
i \varepsilon \psi^{\prime}(x)=A(x) \psi(x)
$$

and let us write $\psi$ as

$$
\psi(x)=\sum_{j \geq 1} c_{j}(x) e^{-i / \varepsilon \int_{x_{0}}^{x} e_{j}\left(x^{\prime}\right) d x^{\prime}} \varphi_{j}(x) .
$$

Since $\left\|K_{0}(x)\right\|$ is integrable, $\lim _{x \rightarrow \pm \infty} c_{j}(x)$ exists (see, e.g., Lemma 3.2 below). Let us now define a set of eigenvectors of $A_{q^{*}}(x)$ by choosing

$$
\varphi_{j}^{\star}(-\infty) \equiv \varphi_{q^{*}, j}(-\infty):=\varphi_{j}(-\infty)
$$

and setting

$$
\varphi_{j}^{\star}(x)=W_{q^{*}}(x,-\infty) \varphi_{j}(-\infty) .
$$


We can also write $\psi(x)$ as $\left(e_{j}^{\star} \equiv e_{q^{\star}, j}\right)$

$$
\begin{aligned}
\psi(x) & =\sum_{j \geq 1} c_{j}^{\star}(x) e^{-i / \varepsilon \int_{x_{0}}^{x} e_{j}^{*}\left(x^{\prime}\right) d x^{\prime}} \varphi_{j}^{\star}(x) \\
& =\sum_{j \geq 1} c_{j}^{\star}(x) e^{-i / \varepsilon \int_{x_{0}}^{x} e_{j}\left(x^{\prime}\right) d x^{\prime}} e^{-i / \varepsilon \int_{x_{0}}^{x}\left(e_{j}^{\star}\left(x^{\prime}\right)-e_{j}\left(x^{\prime}\right)\right) d x^{\prime}} \varphi_{j}^{\star}(x) .
\end{aligned}
$$

From (2.39), (2.42), and $\lim _{x \rightarrow-\infty}\left\|P_{q^{*}, j}(x)-P_{j}(x)\right\|=0$ we have

$$
\lim _{x \rightarrow-\infty} e^{+i / \varepsilon \int_{x_{0}}^{x} e_{j}\left(x^{\prime}\right) d x^{\prime}} P_{j}(x) \psi(x)=c_{j}(-\infty) \varphi_{j}(-\infty)
$$

$$
=e^{-i / \varepsilon \int_{x_{0}}^{-\infty}\left(e_{j}^{\star}\left(x^{\prime}\right)-e_{j}\left(x^{\prime}\right)\right) d x^{\prime}} c_{j}^{\star}(-\infty) \varphi_{j}(-\infty)
$$

On the other hand, with the definitions $W_{q}( \pm \infty, \mp \infty)=W_{q}\left( \pm \infty, x_{0}\right) W_{q}\left(x_{0}, \mp \infty\right), 0 \leq$ $q \leq q^{\star}$, we have

$$
\begin{aligned}
\varphi_{j}^{\star}(\infty) & =W_{q^{\star}}(\infty,-\infty) \varphi_{j}(-\infty) \\
& =W_{q^{\star}}(\infty,-\infty) W_{0}\left(-\infty, x_{0}\right) \varphi_{j}\left(x_{0}\right) \\
& =W_{q^{\star}}(\infty,-\infty) W_{0}(-\infty, \infty) \varphi_{j}(+\infty) \\
& \equiv e^{-i \beta_{j}^{\star}} \varphi_{j}(+\infty)
\end{aligned}
$$

the last equality defining the factor $e^{-i \beta_{j}^{*}}$ where $\beta_{j}^{\star}$ is in general complex. Thus, similarly,

$$
e^{-i / \varepsilon \int_{x_{0}}^{+\infty}\left(e_{j}^{*}\left(x^{\prime}\right)-e_{j}\left(x^{\prime}\right)\right) d x^{\prime}} e^{-i \beta_{j}^{*}} c_{j}^{\star}(\infty)=c_{j}(\infty) .
$$

Let $\psi$ be a solution of $(2.38)$ characterized by $c_{j}(-\infty)=1$ and $c_{k}(-\infty)=0$ for $k \neq j$ which solution of $\psi(x)$ is obtained by replacing $c_{j}^{\star}(x)$ by $c_{j}^{\star}\left(x_{0}\right)$ in (2.42), and we have

$$
\sup _{x \in \mathbb{R}}\left|c_{j}^{\star}(x)-c_{j}^{\star}\left(x_{0}\right)\right|=O\left(e^{-\kappa \varepsilon^{-1}}\right), \quad j=1, \ldots, n .
$$

\section{Therefore}

$$
c_{k}(+\infty)=O\left(e^{-\kappa \varepsilon^{-1}}\right), \quad k \neq j
$$

and

$$
c_{j}(\infty)=e^{-i \beta_{j}^{*}} e^{-i / \varepsilon \int_{-\infty}^{+\infty}\left(e_{j}^{*}\left(x^{\prime}\right)-e_{j}\left(x^{\prime}\right)\right) d x^{\prime}}+O\left(e^{-\kappa \varepsilon^{-1}}\right) .
$$

The matrix $S$ defined in the introduction is then given by the following corollary. Corollary 2.5 .

$$
S_{k j}=e^{-i \beta_{j}^{*}} e^{-i / \varepsilon \int_{-\infty}^{+\infty}\left(e_{j}^{*}\left(x^{\prime}\right)-e_{j}\left(x^{\prime}\right)\right) d x^{\prime}} \delta_{k j}+O\left(e^{-\kappa \varepsilon^{-1}}\right) .
$$

Remark. It should be recalled that we did not write explicitly the $\varepsilon$-dependence of $e_{j}^{\star}$ or $P_{q^{*}, j}$, but in Corollary 2.5 we have $\beta_{j}^{\star}=\beta_{j}^{\star}(\varepsilon)$ and $e_{j}^{\star}\left(x^{\prime}\right)=e_{j}^{\star}\left(x^{\prime}, \varepsilon\right)$.
3. Asymptotics of the nondiagonal part of the matrix $S$.

3.1. Stokes lines. ${ }^{\prime}$ From now on, we deal with the case $\mathcal{H}=\mathbf{C}^{2}$; we compute in this section an asymptotic expression for $S_{21}$, which, in the simplest case, reads

$$
S_{21}=e^{-i \theta^{*}(\varepsilon)} e^{-i \gamma^{*}(\varepsilon) \varepsilon^{-1}}\left(1+O\left(e^{-\kappa \varepsilon^{-1}}\right)\right)
$$

The idea is to combine our iterative scheme $(2.11),(2.12)$ with an analysis in the complex plane by a method due to Fröman and Fröman [1]. To perform the analysis we need some precise information about the analytic extension of $A(x)$ into the complex plane. In particular, we must control the Stokes lines of the problem (Condition II below). Thus, in this subsection we introduce the notion of Stokes lines and give the conditions needed to make use of the method of [1] in the next subsection.

Without restricting the generality we impose $\operatorname{tr} A(x) \equiv 0$. Thus we have $A(x)^{2}=$ $\rho(x) 1$, with this identity defining the function $\rho(x)$. The eigenvalues of $A(x)$ are then $e_{1}(x)=-e_{2}(\dot{x})$ and $e_{2}(x)=\sqrt{\rho(x)}$, with $\sqrt{1}=1$.

The corresponding eigenprojections are given by

$$
P_{j}(x)=\frac{1}{2}\left(1+\frac{A(x)}{e_{j}(x)}\right) .
$$

On $\mathbf{R}$ the eigenvalues are real and distinct and we suppose that there exists $g>0$ with $\rho(x)>g$, for all $x \in \mathbf{R}$.

Let $\Omega$ be a domain of $\mathbf{C}$, symmetric with respect to the real axis, containing $\mathbf{R}$, on which $A$ has an analytic extension. Since $\rho$ is real on $\mathbf{R}$ we have for any $z \in \Omega, \rho(\bar{z})=\overline{\rho(z)}$. The analysis of $S_{21}$ is done by working in the upper half-plane only, whereas the analysis of $S_{12}$ is performed in the lower half-plane, as we shall see below. The eigenvalues and eigenprojections also have analytic extensions in $\Omega$, but it is clear that the zeros of $\rho$ in $\Omega$ are singular points for these objects. Some of these singularities play a dominant role in the determination of $S_{j k}, j \neq k$.

As in $\S 2$ we introduce new operators $A_{q}(z)$ for all $z \in \Omega \backslash\left\{z^{\prime}: \rho\left(z^{\prime}\right)=0\right\}$ by the iteration scheme (2.11) and (2.12). In our case we can write

$$
\begin{aligned}
K_{0}(z) & =P_{1}^{\prime}(z) P_{1}(z)+P_{2}^{\prime}(z) P_{2}(z) \\
& =\left[P_{1}^{\prime}(z), P_{1}(z)\right]=\frac{1}{4 \rho(z)}\left[A^{\prime}(z), A(z)\right],
\end{aligned}
$$

where' $=\frac{d}{d z}$ and we compute for all $q$

$$
\begin{aligned}
A_{q}^{\prime}(z) & =A(z)-i \varepsilon\left[P_{q-1,1}^{\prime}(z), P_{q-1,1}(z)\right] \\
& =A(z)-\frac{i \varepsilon}{4 \rho_{q-1}(z)}\left[A_{q-1}^{\prime}(z), A_{q-1}(z)\right] .
\end{aligned}
$$

Indeed, we have $\operatorname{tr} A_{q^{\prime}}(z) \equiv 0$, because the trace of a commutator is zero. Thus $\rho_{q^{\prime}}(z)$ is defined by $A_{q^{\prime}}^{2}(z)=\rho_{q^{\prime}}(z) 1$. Hence the eigenvalues $e_{q^{\prime}, j}(z)=(-1)^{j} \sqrt{\rho_{q^{\prime}}(z)}$ and $P_{q^{\prime}, 1}(z)$ is given by an expression similar to (3.2). Equation (3.4) clearly shows that although the eigenvectors and eigenprojections are multivalued in $\Omega$ when we perform the analytic continuation, this is not the case for $A_{q}(z)$. In the above construction we must avoid the zeros of $\rho_{q^{\prime}}(z)$ for $q^{\prime} \leq q-1$.

Condition I. The set $X=\{z \in \Omega: \rho(z)=0\}$ is a finite set. Let $r_{2}>0$ such that $D\left(z_{j} ; r_{2}\right) \cap D\left(z_{k} ; r_{2}\right)=\varnothing$ for all $z_{j} \neq z_{k} \in X$ and let

$$
\tilde{\Omega}=\Omega \backslash \bigcup_{z_{j} \in X} D\left(z_{j} ; r_{2}\right) \text {. }
$$


There exist constants $g^{\prime}>0$ and $C^{\prime}<\infty$ such that uniformly on $\tilde{\Omega}$

$$
|\rho(z)| \geq g^{\prime}, \quad\left\|P_{j}(z)\right\| \leq C^{\prime} .
$$

Remark. As we shall see in Conditions II and III below, we must satisfy (3.6) on a subset of $\tilde{\Omega}$ only

Condition I allows us to verify the hypotheses of Lemma 2.1 uniformly on $\tilde{\Omega}$. Moreover the operators $A_{q}(z)$ are holomorphic on $\tilde{\Omega}$, provided $\varepsilon$ is small enough Indeed for any $\varepsilon \leq \varepsilon^{\star}$ and $q \leq q^{\star}$

$$
\rho_{q}(z)=\rho(z)+O\left(b \varepsilon^{2}\right) .
$$

(The proof is the same as that of Corollary 2.2.) We define eigenvectors of $A_{q^{*}}(z), z \in$ $\Omega$, by the method of $\S 2$. Let $\varphi_{j}^{\star}(0)$ be an eigenvector of $A_{q^{\star}}(0)$ for the eigenvalue $e_{j}^{\star}(0), j=1,2$. Let $W_{\star}(z \mid \alpha)$ be the analytic continuation of $W_{\star}(x, 0)$ along a path $\alpha$ in $\tilde{\Omega}$, starting at 0 and ending at $z$, where

$$
W_{\star}^{\prime}(x, 0)=K_{q^{\star}}(x) W_{\star}(x, 0), \quad x \in \mathbf{R},
$$

$$
W_{\star}(0,0)=1 \text {. }
$$

The operator $W_{\star}(z \mid \alpha)$ is a (local) solution of

$$
W_{\star}^{\prime}(z \mid \alpha)=K_{q^{\star}}(z) W_{\star}(z \mid \alpha) .
$$

The main property of $W_{\star}(z \mid \alpha)$, which follows from (3.9) (see (2.13) and (2.14)), is that the vectors

$$
\varphi_{j}^{\star}(z \mid \alpha) \equiv W_{\star}(z \mid \alpha) \varphi_{j}^{\star}(0), \quad j=1,2
$$

are two eigenvectors of $A_{q^{*}}(z)$, which are obtained by analytical continuation of $\varphi_{j}^{\star}(0)$ along $\alpha$. The vector $\varphi_{j}^{\star}(z \mid \alpha)$ is an eigenvector for the eigenvalue $e_{j}^{\star}(z \mid \alpha)$, which is the analytic continuation of $e_{j}^{\star}(0)$ along $\alpha$.

LEMMA 3.1. Let $z_{j}$ be a simple zero of $\rho$ in $\Omega$ and let $\eta$ be a simple closed path around $D\left(z_{j} ; r_{2}\right)$, counterclockwise oriented and encircling no other disc $D\left(z_{k} ; r_{2}\right)$ with $\rho\left(z_{k}\right)=0$. Then for $\varepsilon$ small enough,

1) the total variation of the argument of $\rho_{q^{*}}$ along $\eta$ is $2 \pi$, and

2) if $\eta$ starts at $z=0$, then there exist two complex numbers $\theta_{j k}^{\star}, j \neq k \quad j, k=1,2$; such that

and

$$
W_{\star}(0 \mid \eta) \varphi_{k}^{\star}(0):=e^{i \theta_{j k}^{\star}} \varphi_{j}^{\star}(0), \quad j \neq k
$$

$$
e^{i \theta_{k j}^{*}} e^{i \theta_{j k}^{*}}=-1, \quad j \neq k .
$$

Proof. 1) Using (3.7), we can write

$$
\rho_{q^{*}}(z)=\rho(z) g(z)
$$

with $|g(z)-1|<1$ for all $z \in \eta$. Thus

$$
\begin{aligned}
0=\frac{1}{2 \pi i} \int_{\eta} \frac{g^{\prime}(z)}{g(z)} d z & =\frac{1}{2 \pi i} \int_{\eta} \frac{\rho_{q^{*}}^{\prime}(z)}{\rho_{q^{*}}(z)} d z-\frac{1}{2 \pi i} \int_{\eta} \frac{\rho^{\prime}(z)}{\rho(z)} d z \\
& =\frac{1}{2 \pi i} \int_{\eta} \frac{\rho_{q^{*}}^{\prime}(z)}{\rho_{q^{*}}(z)} d z-1 .
\end{aligned}
$$

2) $\varphi_{j}^{\star}(0)$ is an eigenvector of $A_{q^{*}}(0)$ for the eigenvalue $e_{j}^{\star}(0)$. After analytical continuation $e_{j}^{\star}(0 \mid \eta)$ is an eigenvalue of $A_{q^{*}}(0)$ and by 1$)$ it is equal to $-e_{j}^{\star}(0)=$ $e_{k}^{\star}(0), k \neq j$. Thus $\varphi_{j}^{\star}(0 \mid \eta) \equiv W_{\star}(0 \mid \eta) \varphi_{j}^{\star}(0)$ is an eigenvector for the eigenvalue $e_{k}^{\star}(0)$ and therefore proportional to $\varphi_{k}^{\star}(0)$. Finally, the last identity is a consequence of $\operatorname{det} W_{\star}(z \mid \alpha)=1$ since $\operatorname{tr} K_{q^{*}}(z) \equiv 0$.

Let $\Sigma$ be a simply connected domain in $\tilde{\Omega}$, which contains the real axis. In $\Sigma$ the analytic continuations of $e_{j}^{\star}(x)$ and $\varphi_{j}^{\star}(x)$ are path independent so that we write $e_{j}^{\star}(z)$ instead of $e_{j}^{\star}(z \mid \alpha)$ and so on. Let $\psi(z)$ be a solution of

$$
i \varepsilon \psi^{\prime}(z)=A(z) \psi(z), \quad z \in \Sigma .
$$

We decompose $\psi(z)$ along the eigenvectors of $A_{q^{\star}}(z)$,

$$
\psi(z)=\sum_{j=1}^{2} c_{j}^{\star}(z) e^{-i / \varepsilon \int_{0}^{z} e_{j}^{\star}\left(z^{\prime}\right) d z^{\prime}} \varphi_{j}^{\star}(z),
$$

and we derive a differential equation for the unknown coefficients $c_{j}^{\star}(z)$ using the identities

$$
A(z)=A_{q^{\star}}(z)+i \varepsilon K_{q^{\star}-1}(z)
$$

and

$$
\varphi_{j}^{\star \prime}(z)=K_{q^{\star}}(z) \varphi_{j}^{\star}(z)
$$

By performing scalar products with $W_{\star}^{-1}(z)^{\dagger} \varphi_{j}^{\star}(0), j=1,2$, where $\dagger$ denotes the adjoint, we get a set of linear equations to be solved for $c_{j}^{\star \prime}(z)$. Let $R$ be the constant matrix defined by

$$
R=\left(\begin{array}{ll}
\left\langle\varphi_{1}^{\star}(0) \mid \varphi_{1}^{\star}(0)\right\rangle & \left\langle\varphi_{1}^{\star}(0) \mid \varphi_{2}^{\star}(0)\right\rangle \\
\left\langle\varphi_{2}^{\star}(0) \mid \varphi_{1}^{\star}(0)\right\rangle & \left\langle\varphi_{2}^{\star}(0) \mid \varphi_{2}^{\star}(0)\right\rangle
\end{array}\right)^{-1}
$$

the elements of which, denoted by $r_{j k}$, are $O(1)$. We obtain finally

$$
c_{j}^{\star \prime}(z)=\sum_{k=1}^{2} \exp \left(i \varepsilon^{-1} \Delta_{j k}^{\star}(z)\right) a_{j k}(z) c_{k}^{\star}(z),
$$

where

$$
\Delta_{j k}^{\star}(z)=\int_{0}^{z}\left(e_{j}^{\star}\left(z^{\prime}\right)-e_{k}^{\star}\left(z^{\prime}\right)\right) d z^{\prime}
$$

and

$$
a_{j k}(z)=-\sum_{l=1}^{2} r_{j l}\left\langle\varphi_{l}^{\star}(0) \mid W_{\star}^{-1}(z)\left(K_{q^{*}}(z)-K_{q^{\star}-1}(z)\right) W_{\star}(z) \varphi_{k}^{\star}(0)\right\rangle
$$

We have a good control of $a_{j k}(z)$ using Lemma 2.1 but the factor $\exp \left(i \varepsilon^{-1} \Delta_{j k}^{\star}(z)\right)$ may cause trouble when we consider the limit $\varepsilon \rightarrow 0$ because $\operatorname{Im} \Delta_{j k}^{\star}(z) \neq 0$. Since $e_{j}^{\star}(z)=e_{j}(z)+O\left(\varepsilon^{2} b\right)$, we must actually control the factor $\exp \left(i \varepsilon^{-1} \Delta_{j k}(z)\right)$, where

$$
\Delta_{j k}(z)=\int_{0}^{z}\left(e_{j}\left(z^{\prime}\right)-e_{k}\left(z^{\prime}\right)\right) d z^{\prime}
$$




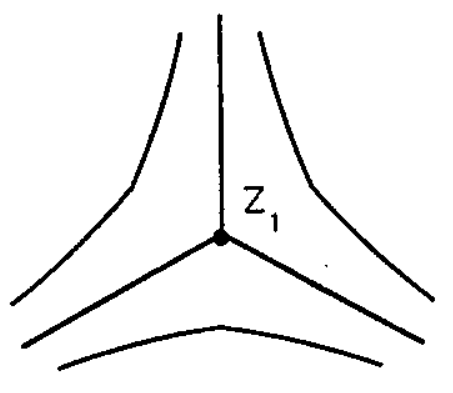

Frg. 1. The level lines of $\Phi(z)$ near $z_{0}$.
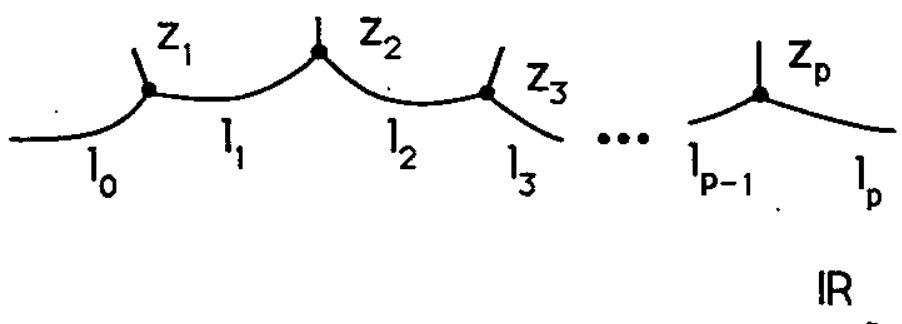

FIG. 2. The Stokes lines of Condition II.

The function $\Delta_{j k}$ is equal, up to a factor \pm 2 , to the function

$$
\Phi(z):=\int_{0}^{z} \sqrt{\rho\left(z^{\prime}\right)} d z^{\prime},
$$

which is naturally associated with the quadratic differential $\rho(z) d^{2} z$

DEFINITION. A Stokes line $\alpha$ is a curve in $\Omega \backslash\{z: \rho(z)=0\}$ such that

1) $\operatorname{Im} \Phi(z)$ is a constant along $\alpha$,

2) $\alpha$ is maximal with property 1 ), and

3) one of the boundary points of $\alpha$ at least is a zero of $\rho(z)$.

There are different terminologies in the literature. Sometimes our Stokes lines are called antiStokes lines and vice versa (see below). A Stokes line is always a simple curve and in our case it is contained either in the upper half-plane or in the lower half-plane. Near a simple zero $z_{0}$ of $\rho(z)$ the level-lines of $\operatorname{Im} \Phi(z)$ are homeomorphic to the level-lines

$$
\operatorname{Im} z^{3 / 2}=\text { constant }
$$

around $z=0$. For any simple zero $z_{0}$ of $\rho(z)$ there are exactly three Stokes lines which have $z_{0}$ as boundary point. We call them the Stokes lines of $z_{0}$ (see Fig. 1)

CoNDITION II. A) There exists in the upper half-plane a nonempty finite set of simple zeros of $\rho(z),\left\{z_{1}, \ldots, z_{p}\right\}$ with the following properties (see Fig. 2):

1) There exists a Stokes line $l_{i}$, parameterized by $\left(t_{i}, t_{i+1}\right)$, such that $\lim _{t \rightarrow t_{i}} l_{i}(t)=z_{i}, \lim _{t \rightarrow t_{i+1}} l_{i}(t)=z_{i+1}, i=1, \ldots, p-1$

2) There exists a Stokes line $l_{0}$, parameterized by $\left(-\infty, t_{1}\right)$, such that $\lim _{t \rightarrow t_{1}} l_{0}(t)=z_{1}, \lim _{t \rightarrow-\infty} \operatorname{Re} l_{0}(t)=-\infty, \lim _{t \rightarrow-\infty} \operatorname{Im} l_{0}(t)=a^{-}$

3) there exists a Stokes line $l_{p}$, parameterized by $\left(t_{p}, \infty\right)$, such that $\lim _{t \rightarrow t_{p}} l_{p}(t)=z_{p}, \lim _{t \rightarrow \infty} \operatorname{Re} l_{p}(t)=\infty, \lim _{t \rightarrow \infty} \operatorname{Im} l_{p}(t)=a^{+}$.

B) Along any vertical line $\operatorname{Re} z=x$ going from the real axis to $l_{0}$ or $l_{p}, \operatorname{Im} \Phi(z)$ is strictly monotone, provided $|x|$ is large enough.

Remark. Condition II describes the situation illustrated in Fig. 2.

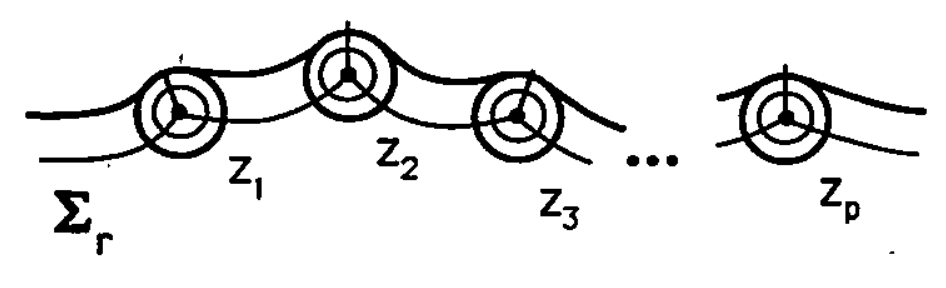

$\mathbb{R}$

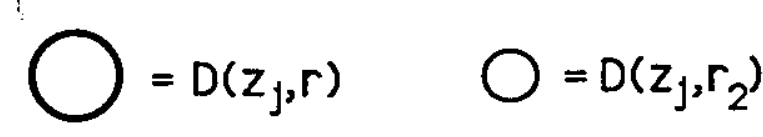

FIG. 3. The set $\Sigma_{\mathrm{r}}$ of Condition III.

In our case, if Condition II is satisfied then an analogous condition holds in the lower half-plane. It follows from Theorem 2.1 in [7] that the region $\Lambda$ in the upper half-plane between the real axis and the closure of the Stokes lines $l_{0}, \ldots, l_{p}$ is a simply connected region in $\Omega$ which does not contain zeros of $\rho$ in its interior. In [7], part B of Condition II follows from the existence of limiting matrices when $t$ tends to infinity. As already noted, such limiting matrices are not supposed to exist here. Let $r>0$ and let

$$
\Sigma_{r}=\left\{z \in \mathbf{C} \mid \operatorname{dist}(z, \Lambda) \leq r \text { and }\left|z-z_{i}\right| \geq r, i=1, \ldots, p\right\} .
$$

CONDITION III. There exists $r>r_{2}$, sufficiently small so that $\Sigma_{r}$ is a simply connected region in $\Omega$ containing the real axis and such that, for any zero $z_{i}, i=$ $1, \ldots, p$, each Stokes line of $z_{i}$ in the disc $D\left(z_{i} ; r\right)$ intersects the boundary of the disc at a single point, $D\left(z_{i}, r\right) \cap D\left(z_{j}, r\right)=\varnothing$ (see Fig. 3).

The function

$$
b(x):=\sup _{y:}\left\|K_{0}(x+i y)\right\|
$$

tends to zero at infinity and is integrable on $\mathbf{R}$.

Remark. As we already mentioned, we need to verify Condition I on $\Sigma_{\tau}$ only and not on $\tilde{\Omega}$ since we shall integrate the differential equation (3.18) along a path in $\Sigma_{r}$.

3.2. The Fröman-Fröman method. We suppose that Conditions I-III are satisfied and we study equation (3.18) on $\Sigma_{r}$. The hypotheses of Lemma 2.1 are thus verified uniformly on $\Sigma_{r}$, so that there exists a $q^{\star}=q^{\star}(\varepsilon)$ independent of $z \in \Sigma_{r}$ provided $\varepsilon$ is small enough. Let us rewrite equation (3.18) as a Volterra equation

$$
c_{1}^{\star}(z)=c_{1}^{\star}\left(z_{0}\right)+\int_{z_{0}}^{z} a_{11}\left(z^{\prime}\right) c_{1}^{\star}\left(z^{\prime}\right) d z^{\prime}+\int_{z_{0}}^{z} a_{12}\left(z^{\prime}\right) e^{i \varepsilon^{-1} \Delta_{12}^{*}\left(z^{\prime}\right)} c_{2}^{\star}\left(z^{\prime}\right) d z^{\prime}
$$

and

(3.27) $\quad c_{2}^{\star}(z)=c_{2}^{\star}\left(z_{0}\right)+\int_{z_{0}}^{z} a_{22}\left(z^{\prime}\right) c_{2}^{\star}\left(z^{\prime}\right) d z^{\prime}+\int_{z_{0}}^{z} a_{21}\left(z^{\prime}\right) e^{i \varepsilon^{-1} \Delta_{21}^{\star}\left(z^{\prime}\right)} c_{1}^{\star}\left(z^{\prime}\right) d z^{\prime}$.

LEMMA 3.2. If Conditions I-III hold then $\lim _{x \rightarrow \pm \infty} c_{j}^{\star}(x)=c_{j}^{\star}( \pm \infty)$ exist and

$$
\lim _{x \rightarrow \pm \infty} \sup _{y=t}\left|c_{j}^{\star}(x+i y)-c_{j}^{\star}( \pm \infty)\right|=0 .
$$




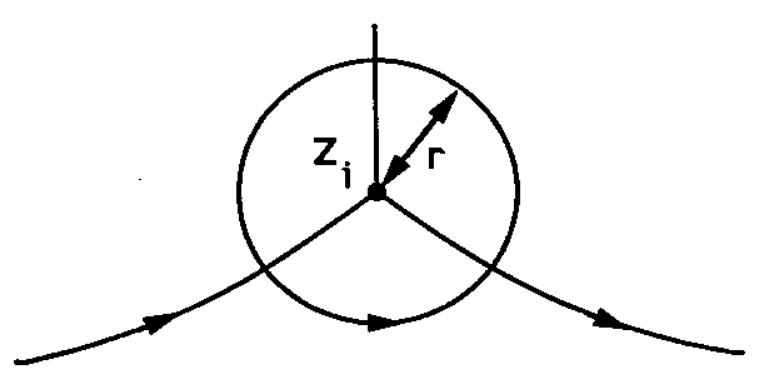

FiG. 4. The path of integration close to $z_{i}$.

Proof. By Conditions I-III we get from (3.20) and Lemma 2.1, as in $\S 2$,

$$
\sup _{y=}\left|a_{k j}(x+i y)\right|=b(x) O\left(e^{-\kappa \varepsilon^{-1}}\right)
$$

and for all $z \in \Sigma_{r}$

$$
\Delta_{j k}^{\star}(z)=\Delta_{j k}(z)+O\left(\varepsilon^{2}\right)
$$

Hence the limits $\lim _{x \rightarrow \pm \infty} c_{j}^{\star}(x)$ exist on the real axis since $\Delta_{j k}$ is real there. Then for all $z=x+i y$ on a vertical segment joining $\mathbf{R}$ and $l_{0}$ or $l_{p}$ we can control $\left|\operatorname{Im} \Delta_{j k}(z)\right|$, provided $|x|$ is large enough, using part B of Condition II. Indeed, for such $z,\left|\operatorname{Im} \Delta_{j k}(z)\right|$ is bounded by twice the value of $|\operatorname{Im} \Phi(z)|$ on the Stokes lines. From these estimates and (3.28) we can easily deduce Lemma 3.2 using (3.26) and From these est

Instead of integrating (3.18) along the real axis we integrate the equation along the Stokes lines $l_{0}, \ldots, l_{p}$, as long as we are at a distance larger than $r$ from a zero of $\rho$. Otherwise we integrate the equation along the boundaries of the discs $D\left(z_{i} ; r\right)$, staying always in $\Sigma_{r}$ (see Fig. 4).

Let $z$ and $z_{0}$ be two points of $\Sigma_{r}$ and let $T\left(z, z_{0}\right)$ be the matrix-solution of (3.18) with $T\left(z_{0}, z_{0}\right)=1$. We can find $T\left(z, z_{0}\right)$ by integrating the equation along any path in $\Sigma_{r}$ going from $z_{0}$ to $z$. However, because of the factors $\exp \left(i \varepsilon^{-1} \Delta_{j k}(z)\right)$ we have a good control of the equation only on particular paths. For instance, the Stokes lines are "good" paths. The main work consists of controlling the equation along the parts of the boundaries of the dises $D\left(z_{i} ; r\right)$ when we pass from one Stokes line to the next.

LEMMA 3.3. Let $z$ and $z_{0} \in \Sigma_{r}$ and let $\alpha$ be a path, parameterized by $\left[s_{0}, s_{1}\right]$, going from $z_{0}$ to $z$, and such that $s \mapsto \operatorname{Im} \Delta_{12}(\alpha(s))$ is nondecreasing on $\left[s_{0}, s_{1}\right]$. Then

$$
T\left(z, z_{0}\right)=\left(\begin{array}{cc}
1+O\left(e^{-\kappa \varepsilon^{-1}}\right) & e^{-\varepsilon^{-1} \operatorname{Im} \Delta_{12}^{*}\left(z_{0}\right)} O\left(e^{-\kappa \varepsilon^{-1}}\right) \\
e^{\varepsilon^{-1} \operatorname{Im} \Delta_{12}^{*}(z)} O\left(e^{-\kappa \varepsilon^{-1}}\right) & +O\left(e^{-\kappa \varepsilon^{-1}}\right) \\
& +O\left(e^{-2 \kappa \varepsilon^{-1}}\right) e^{\varepsilon^{-1}\left(\operatorname{Im} \Delta_{12}^{*}(z)-\operatorname{Im} \Delta_{12}^{*}\left(z_{0}\right)\right)}
\end{array}\right) .
$$

Proof. We consider (3.26) and (3.27) along $\alpha$ with $c_{1}^{\star}\left(z_{0}\right)=1$ and $c_{2}^{\star}\left(z_{0}\right)=0$ and we introduce new variables
(3.30)
$X_{1}(s)=c_{1}^{\star}(\alpha(s))$,
$X_{2}(s)=e^{i \varepsilon^{-1} \Delta_{12}^{*}(\alpha(s))} c_{2}^{\star}(\alpha(s))$.

Writing $b_{j k}(s)=a_{j k}(\alpha(s)) \frac{d \alpha(s)}{d s}$ and $\Delta_{12}^{*}(s) \equiv \Delta_{12}^{*}(\alpha(s))$, we get

(3.31) $\quad X_{1}(s)=1+\int_{s_{0}}^{s} b_{11}\left(s^{\prime}\right) X_{1}\left(s^{\prime}\right) d s^{\prime}+\int_{s_{0}}^{s} b_{12}\left(s^{\prime}\right) X_{2}\left(s^{\prime}\right) d s^{\prime}$

$$
X_{2}(s)=\int_{s_{0}}^{s} b_{22}\left(s^{\prime}\right) e^{i \varepsilon^{-1}\left(\Delta_{12}^{*}(s)-\Delta_{12}^{*}\left(s^{\prime}\right)\right)} X_{2}\left(s^{\prime}\right) d s^{\prime}
$$

$$
+\int_{s_{0}}^{s} b_{21}\left(s^{\prime}\right) e^{i \varepsilon^{-1}\left(\Delta_{12}^{*}(s)+\Delta_{21}^{\star}\left(s^{\prime}\right)\right)} X_{1}\left(s^{\prime}\right) d s^{\prime}
$$
In (3.32) $s^{\prime} \leq s$ and $\Delta_{21}^{\star}\left(s^{\prime}\right)=-\Delta_{12}^{\star}\left(s^{\prime}\right)$. Using (3.29) and the hypothesis on the path

$$
\left|e^{i \varepsilon^{-1}\left(\Delta_{12}^{*}(s)-\Delta_{12}^{*}\left(s^{\prime}\right)\right)}\right|
$$$$
=\exp \left(-\varepsilon^{-1}\left(\operatorname{Im} \Delta_{12}(s)-\operatorname{Im}\left(\Delta_{12}\left(s^{\prime}\right)\right)\right)+O(\varepsilon)\right)=O(\exp (O(\varepsilon))) .
$$
$p_{s_{0} \leq s \leq s_{1}}\left|X_{i}(s)\right|$. We get from (3.31), (3.32), and (3.33), using (3.28),

$$
\begin{aligned}
& \left\|X_{1}\right\| \leq 1+O\left(e^{-\kappa \varepsilon^{-1}}\right)\left(\left\|X_{1}\right\|+\left\|X_{2}\right\|\right) \\
& \left\|X_{2}\right\| \leq O\left(e^{-\kappa \varepsilon^{-1}}\right)\left(\left\|X_{1}\right\|+\left\|X_{2}\right\|\right)
\end{aligned}
$$

so that for $\varepsilon$ small enough $\left\|X_{1}\right\|+\left\|X_{2}\right\| \leq 2$. Using this a priori estimate in (3.31) and (3.32) we have

$$
\sup _{s_{0} \leq s \leq s_{1}}\left|X_{1}(s)-1\right|=O\left(e^{-\kappa \varepsilon^{-1}}\right)
$$

and

$$
\sup _{s_{0} \leq s \leq s_{1}}\left|X_{2}(s)\right|=O\left(e^{-\kappa \varepsilon^{-1}}\right)
$$

Equations (3.35) and (3.36) allow us to determine the first column of $T\left(z, z_{0}\right)$,

$$
T\left(z, z_{0}\right)=\left(\begin{array}{cc}
1+O\left(e^{-\kappa \varepsilon^{-1}}\right) & T_{12}\left(z, z_{0}\right) \\
e^{\varepsilon^{-1} \operatorname{Im} \Delta_{\mathbf{1} 2}^{*}(z)} O\left(e^{-\kappa \varepsilon^{-1}}\right) & T_{22}\left(z, z_{0}\right)
\end{array}\right)
$$

Since $\left|a_{11}(z)+a_{22}(z)\right|=O\left(e^{-\kappa \varepsilon^{-1}}\right)$, we get from the Liouville formula

$$
\begin{aligned}
\operatorname{det} T\left(z, z_{0}\right) & =\exp \left(O\left(e^{-\kappa \varepsilon^{-1}}\right)\right) \\
& =1+O\left(e^{-\kappa \varepsilon^{-1}}\right)
\end{aligned}
$$

Moreover $T^{-1}\left(z, z_{0}\right)=T\left(z_{0}, z\right)$, hence

$$
T\left(z_{0}, z\right)=\frac{1}{\operatorname{det} T\left(z, z_{0}\right)}\left(\begin{array}{cc}
T_{22}\left(z, z_{0}\right) & -T_{12}\left(z, z_{0}\right) \\
-T_{21}\left(z, z_{0}\right) & T_{11}\left(z, z_{0}\right)
\end{array}\right)
$$

The reverse path $\alpha^{-1}$ from $z$ to $z_{0}$ is such that $s \mapsto \operatorname{Im} \Delta_{21}\left(\alpha^{-1}(s)\right)$ is nonincreasing from $s_{1}$ to $s_{0}$. If $c_{1}^{\star}(z)=0$ and $c_{2}^{\star}(z)=1$ then we can estimate $c_{1}^{\star}\left(z_{0}\right)$ and $c_{2}^{\star}\left(z_{0}\right)$ as above, introducing new variables $Y_{2}(s)=c_{2}^{\star}\left(\alpha^{-1}(s)\right)$ and $Y_{1}(s)=e^{i \varepsilon^{-1} \Delta_{21}^{\star}\left(\alpha^{-1}(s)\right)} \times$ $c_{1}^{\star}\left(\alpha^{-1}(s)\right)$. Thus we can estimate the second column of (3.39). The coefficient $T_{22}\left(z, z_{0}\right)$ is estimated using $\operatorname{det} T\left(z, z_{0}\right)=1+O\left(e^{-\kappa \varepsilon^{-1}}\right)$.

A Stokes line is a good path because $\operatorname{Im} \Delta_{j k}(z)$ remains constant along this line. The following corollary is thus immediate.

COROLLARY 3.4. If there is a Stokes line going from $z_{0}$ to $z$, then

$$
T\left(z, z_{0}\right)=\left(\begin{array}{cc}
1+O\left(e^{-\kappa \varepsilon^{-1}}\right) & O\left(e^{-\kappa \varepsilon^{-1}}\right) e^{-\varepsilon^{-1} \operatorname{Im} \Delta_{12}^{*}(z)} \\
O\left(e^{-\kappa \varepsilon^{-1}}\right) e^{\varepsilon^{-1} \operatorname{Im} \Delta_{12}^{*}(z)} & 1+O\left(e^{-\kappa \varepsilon^{-1}}\right)
\end{array}\right) .
$$

We now come to the difficult part of the method. We must control the matrix solution $T\left(z, z_{0}\right)$ along a portion of $\partial D\left(z_{j}, r\right)$, which is not a good path in the sense that $\operatorname{Im} \Delta_{12}(z)$ is not monotone. We must establish two lemmas. The first lemma 


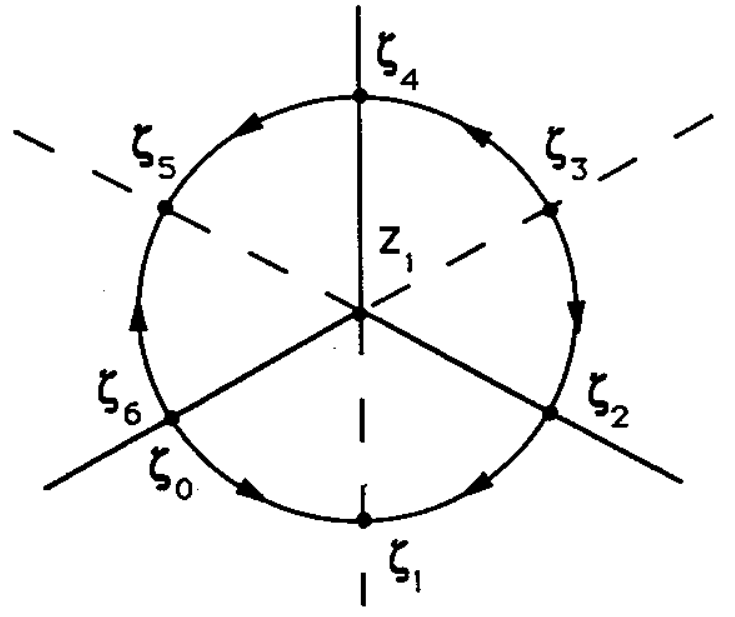

FIG. 5. The points $\zeta_{j}, j=0, \ldots, 6$ on the Stokes and antiStokes lines.

gives a monodromy matrix around the singularity $z_{1}$ and is easily proven. The second and main lemma is more diffeult to establish "Its proof is based on Lemmas 3.3 and 3.5 and on a clever use of $2 \times 2$ matrices and their inverses [1]. This method has a definite advantage over the use of stretching and matching techniques to compute asymptotics in the sense that it allows us to obtain better estimates on the remainders (see (1.19) in the introduction). However, it can only be used for simple zeros of the function $\rho(z)$, whereas the stretching and matching method works in more general situations [24].

We consider now the neighborhood of a zero of $\rho(z)$, say $z_{1}$. Let $\delta$ be the boundary of the disc $D\left(z_{1} ; r\right)$ counterclockwise oriented, going from $\zeta_{0}$ to $\zeta_{6}$ as in Fig. 5 . On this figure the solid lines are the Stokes lines of $z_{1}$ and the dashed lines are the antiStokes lines of $z_{1}$, i.e., the lines along which $\operatorname{Re} \Delta_{12}(z) \equiv \operatorname{Re} \Delta_{12}\left(z_{1}\right)$. The arrows indicate the directions in which $\operatorname{Im} \Delta_{12}(z)$ is nondecreasing along the boundary of $D\left(z_{1} ; r\right)$.

We compute the matrix $T\left(\zeta_{6}, \zeta_{0}\right)$ along $\delta$.

LEMMA 3.5 .

$$
T\left(\zeta_{6}, \zeta_{0}\right)=\left(\begin{array}{cc}
0 & e^{i / \varepsilon \int_{\eta} e_{1}^{\star}} e^{-i \theta_{21}^{*}} \\
e^{i / \varepsilon \int_{\eta} e_{2}^{\star}} e^{-i \theta_{12}^{*}} & 0
\end{array}\right)
$$

Proof. Let us consider $\psi(z)$ at $z=\zeta_{0}$, the solution of which we have obtained by integration along the Stokes line $l_{0}$ up to $\zeta_{0}$. We have

$$
\psi\left(\zeta_{0}\right)=\sum_{j=1}^{2} c_{j}^{\star}\left(\zeta_{0}\right) e^{-i / \varepsilon \int_{0}^{\zeta_{0}} e_{j}^{\star}} \varphi_{j}^{\star}\left(\zeta_{0}\right),
$$

where in (3.40) the integration from 0 to $\zeta_{0}$ is along $\alpha$ as in Fig. 6 and, similarly, $\varphi_{j}^{\star}\left(\zeta_{0}\right)$ is the analytical continuation of $\varphi_{j}^{\star}(0)$ along $\alpha$.

We make the analytical continuation of (3.40) along $\delta$ up to $\zeta_{6}$. Since $\psi(z)$ is holomorphic at $z_{1}$ we have $\psi\left(\zeta_{6}\right)=\psi\left(\zeta_{0}\right)$ and we can write

$$
\psi\left(\zeta_{0}\right)=\sum_{j=1}^{2} c_{j}^{\star}\left(\zeta_{6}\right) e^{-i / \varepsilon \int_{\alpha} e_{j}^{*}} e^{-i / \varepsilon \int_{\delta} e_{j}^{\star}} \varphi_{j}^{\star}\left(\zeta_{6}\right)
$$

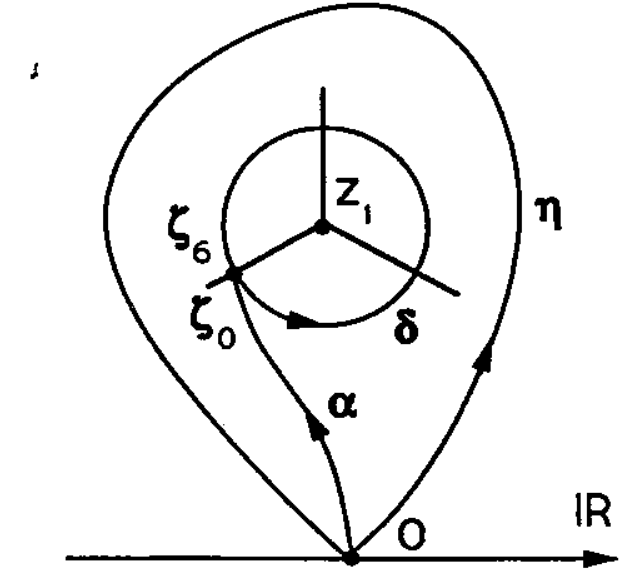

FIG. 6. The paths $\alpha, \delta$, and $\eta$.

where now $\varphi_{j}^{\star}\left(\zeta_{6}\right)$ is the analytical continuation of $\varphi_{j}^{\star}(0)$ along $\alpha$ and then along $\delta$ But this is the same as the analytical continuation of $\varphi_{j}^{\star}(0)$ along $\eta$ and then along $\alpha$ as in Fig. 6. By Lemma 3.1 we therefore have

$$
\varphi_{j}^{\star}\left(\zeta_{6}\right)=e^{i \theta_{k j}^{\star}} \varphi_{k}^{\star}\left(\zeta_{0}\right)
$$

Similarly we have

$$
\int_{\alpha} e_{j}^{\star}+\int_{\delta} e_{j}^{\star}=\int_{\eta} e_{j}^{\star}+\int_{\alpha} e_{k}^{\star} .
$$

Hence, by comparing (3.40) and (3.41),

$$
c_{j}^{\star}\left(\zeta_{6}\right) e^{-i / \varepsilon \int_{\eta} e_{j}^{*}} e^{i \theta_{k j}^{*}}=c_{k}^{\star}\left(\zeta_{0}\right), \quad k \neq j .
$$

LEMMA 3.6. For $\varepsilon$ small enough

$$
T\left(\zeta_{2}, \zeta_{0}\right)=\left(\begin{array}{cc}
1+O\left(e^{-\kappa / \varepsilon}\right) & O\left(e^{-\kappa / \varepsilon}\right) e^{-\varepsilon^{-1} \operatorname{Im} \Delta_{12}\left(\zeta_{0}\right)} \\
e^{-i / \varepsilon \int_{\eta} e_{1}^{*}} e^{-i \theta_{12}^{\star}}\left(1+O\left(e^{-\kappa / \varepsilon}\right)\right) & 1+O\left(e^{-\kappa / \varepsilon}\right)
\end{array}\right) .
$$

Proof. The following computations will involve expressions such as $\varepsilon^{-1} \operatorname{Im} \Delta_{12}^{*}\left(\zeta_{\nu}\right)$ for $\nu=0,2,4,6$. These expressions are almost equal. Indeed

$$
\Delta_{j k}^{\star}(z)=\Delta_{j k}(z)+O\left(\varepsilon^{2}\right)
$$

and for this choice of $\zeta_{\nu}$ we have

(3.46)

$$
\operatorname{Im} \Delta_{12}\left(\zeta_{\nu}\right)=\operatorname{Im} \Delta_{12}\left(z_{1}\right), \quad \nu=0,2,4,6
$$

since these points are on the Stokes lines of $z_{1}$. Hence, in particular,

(3.47) $\quad e^{ \pm \varepsilon^{-1} \operatorname{Im} \Delta_{12}^{\star}\left(\zeta_{\nu}\right)}=O\left(e^{ \pm \varepsilon^{-1} \operatorname{Im} \Delta_{12}\left(z_{1}\right)}\right), \quad \nu=0,2,4,6$.

Finally note that (see Fig. 6)

$$
\int_{\eta} e_{1}^{\star}=\int_{\eta} e_{1}+O\left(\varepsilon^{2}\right)=\Delta_{12}\left(z_{1}\right)+O\left(\varepsilon^{2}\right) .
$$


Let us denote the coefficient $j k$ of the matrix $T\left(\zeta_{\alpha}, \zeta_{\beta}\right)$ by $t_{j k}(\alpha, \beta)$ and consider the identity

$$
T\left(\zeta_{\nu+1}, \zeta_{\nu}\right)=T\left(\zeta_{\nu+1}, \zeta_{\nu+2}\right) T\left(\zeta_{\nu+2}, \zeta_{\nu}\right)
$$

Using (3.38) again

(3.50) $\quad \operatorname{det} T\left(\zeta_{\mu}, \zeta_{\nu}\right)=t_{11}(\mu, \nu) t_{22}(\mu, \nu)-t_{12}(\mu, \nu) t_{21}(\mu, \nu)=1+O\left(e^{-\kappa \varepsilon^{-1}}\right)$

and we obtain for $\nu=0,2$, and 4

(3.51) $\quad t_{11}(\nu+2, \nu)=\frac{t_{11}(\nu+1, \nu)}{t_{11}(\nu+1, \nu+2)}-\frac{t_{12}(\nu+1, \nu+2)}{t_{11}(\nu+1, \nu+2)} t_{21}(\nu+2, \nu)$,

$$
t_{22}(\nu+2, \nu)=\frac{t_{11}(\nu+1, \nu+2)}{t_{11}(\nu+1, \nu)}\left(1+O\left(e^{-\kappa \varepsilon^{-1}}\right)\right)
$$

$$
+\frac{t_{12}(\nu+1, \nu)}{t_{11}(\nu+1, \nu)} t_{21}(\nu+2, \nu)
$$$$
t_{12}(\nu+2, \nu)=\frac{t_{12}(\nu+1, \nu)}{t_{11}(\nu+1, \nu+2)}-\frac{t_{12}(\nu+1, \nu+2)}{t_{11}(\nu+1, \nu)}\left(1+O\left(e^{-\kappa \varepsilon^{-1}}\right)\right)
$$

$$
-\frac{t_{12}(\nu+1, \nu) t_{12}(\nu+1, \nu+2)}{t_{11}(\nu+1, \nu) t_{11}(\nu+1, \nu+2)} t_{21}(\nu+2, \nu) \text {. }
$$

These identities express, in particular, the elements of the matrix $T\left(\zeta_{2}, \zeta_{0}\right)$ as functions of the element $t_{21}(2,0)$ and other matrix elements that we can control by means of Lemma 3.3:

(3.54) $t_{11}(2,0)=1+O\left(e^{-\kappa \varepsilon^{-1}}\right)+O\left(e^{-\kappa \varepsilon^{-1}}\right) e^{-\varepsilon^{-1} \operatorname{Im} \Delta_{12}\left(z_{1}\right)} t_{21}(2,0)$,

(3.55) $t_{22}(2,0)=1+O\left(e^{-\kappa \varepsilon^{-1}}\right)+O\left(e^{-\kappa \varepsilon^{-1}}\right) e^{-\varepsilon^{-1} \operatorname{Im} \Delta_{12}\left(z_{1}\right)} t_{21}(2,0)$

(3.56) $t_{12}(2,0)=O\left(e^{-\kappa \varepsilon^{-1}}\right) e^{-\varepsilon^{-1} \operatorname{Im} \Delta_{12}\left(z_{1}\right)}+\left(O\left(e^{-\kappa \varepsilon^{-1}}\right) e^{-\varepsilon^{-1} \operatorname{Im} \Delta_{12}\left(z_{1}\right)}\right)^{2} t_{21}(2,0)$.

We are thus led to the determination of $t_{21}(2,0)$. Note that these estimates are true for the elements of $T\left(\zeta_{6}, \zeta_{4}\right)$ if we replace the arguments $(2,0)$ by $(6,4)$. Consider now the identity

$$
T\left(\zeta_{3}, \zeta_{2}\right) T\left(\zeta_{2}, \zeta_{0}\right) T\left(\zeta_{0}, \zeta_{6}\right)=T\left(\zeta_{3}, \zeta_{4}\right) T\left(\zeta_{4}, \zeta_{6}\right)
$$

Using Lemma 3.1 and $e_{1}^{\star} \equiv-e_{2}^{\star}$ to compute $T\left(\zeta_{0}, \zeta_{6}\right)=T\left(\zeta_{6}, \zeta_{0}\right)^{-1}$, we obtain for the coefficient 22 of (3.57)

$$
\begin{aligned}
& t_{21}(3,2) t_{11}(2,0) e^{i \theta_{12}^{\star}} e^{i \varepsilon^{-1} \int_{\eta} e_{1}^{*}}+t_{22}(3,2) t_{21}(2,0) e^{i \theta_{12}^{*}} e^{i \varepsilon^{-1} \int_{\eta} e_{1}^{*}} \\
& \quad=t_{21}(3,4) t_{12}(4,6)+t_{22}(3,4) t_{22}(4,6)
\end{aligned}
$$

and for the coefficient 21 of (3.57)

$$
t_{21}(3,2) t_{12}(2,0) e^{i \theta_{21}^{*}} e^{i \varepsilon^{-1} \int_{\eta} e_{2}^{*}}+t_{22}(3,2) t_{22}(2,0) e^{i \theta_{21}^{*}} e^{i \varepsilon^{-1} \int_{\eta} e_{2}^{*}}
$$$$
=t_{21}(3,4) t_{11}(4,6)+t_{22}(3,4) t_{21}(4,6) \text {. }
$$

\section{Lemma 3.3, (3.39), and (3.47) yield}

(3.60) $\quad t_{21}(3,2)=-t_{21}(2,3)\left(1+O\left(e^{-\kappa \varepsilon^{-1}}\right)\right)=O\left(e^{-\kappa \varepsilon^{-1}}\right) e^{\varepsilon^{-1} \operatorname{Im} \Delta_{12}\left(z_{1}\right)}$

(3.61) $\quad t_{21}(3,4)=-t_{21}(4,3)\left(1+O\left(e^{-\kappa \varepsilon^{-1}}\right)\right)=O\left(e^{-\kappa \varepsilon^{-1}}\right) e^{\varepsilon^{-1} \operatorname{Im} \Delta_{12}\left(z_{1}\right)}$,

(3.62) $\quad t_{22}(3,4)=t_{11}(4,3)\left(1+O\left(e^{-\kappa \varepsilon^{-1}}\right)\right)=1+O\left(e^{-\kappa \varepsilon^{-1}}\right)$,

(3.63) $\quad t_{22}(3,2)=t_{11}(2,3)\left(1+O\left(e^{-\kappa \varepsilon^{-1}}.\right)\right)=1+O\left(e^{-\kappa \varepsilon^{-1}}\right)$,

whereas from (3.39) and the remark following (3.56) we have

(3.64) $t_{12}(4,6)=O\left(e^{-\kappa \varepsilon^{-1}}\right) e^{-\varepsilon^{-1} \operatorname{Im} \Delta_{12}\left(z_{1}\right)}+\left(O\left(e^{-\kappa \varepsilon^{-1}}\right) e^{-\varepsilon^{-1} \operatorname{Im} \Delta_{12}\left(z_{1}\right)}\right)^{2} t_{21}(6,4)$

and

(3.65) $\quad t_{22}(4,6)=1+O\left(e^{-\kappa \varepsilon^{-1}}\right)+O\left(e^{-\kappa \varepsilon^{-1}}\right) e^{-\varepsilon^{-1} \operatorname{Im} \Delta_{12}\left(z_{1}\right)} t_{21}(6,4)$.

Now we use (3.58) and the above results to get

$$
t_{21}(2,0) e^{i \theta_{12}^{*}} e^{i \varepsilon^{-1} \int_{\eta} e_{1}^{\star}}=1+O\left(e^{-\kappa \varepsilon^{-1}}\right)+O\left(e^{-2 \kappa \varepsilon^{-1}}\right) e^{-\varepsilon^{-1} \operatorname{Im} \Delta_{12}\left(z_{1}\right)} t_{21}(2,0)
$$

Hence we see that we have to estimate $t_{21}(6,4)$ as well as determine $t_{21}(2,0)$. This is done by performing a similar computation: We estimate $t_{11}(4,6)$ as a function of $t_{21}(6,4)$ as above and we consider equation (3.59). After multiplication by $e^{-i \theta_{21}^{*} \times}$ $e^{-i \varepsilon^{-1} \int_{\eta} e_{2}^{\star}}$ and using

$$
\operatorname{Im} \int_{\eta} e_{2}^{\star}=-\operatorname{Im} \int_{\eta} e_{1}^{\star}
$$

we obtain another equation for $t_{21}(6,4)$ and $t_{21}(2,0)$ :

$$
-t_{21}(6,4) e^{-i \theta_{21}^{*}} e^{-i \varepsilon^{-1} \int_{\eta} e_{2}^{*}}=1+O\left(e^{-\kappa \varepsilon^{-1}}\right)
$$

(3.68) $+O\left(e^{-2 \kappa \varepsilon^{-1}}\right) e^{-\varepsilon^{-1} \operatorname{Im} \Delta_{12}\left(z_{1}\right)} t_{21}(6,4)+O\left(e^{-\kappa \varepsilon^{-1}}\right) e^{-\varepsilon^{-1} \operatorname{Im} \Delta_{12}\left(z_{1}\right)} t_{21}(2,0)$.

Therefore, from (3.66) and (3.68) we deduce the a priori estimates

$(3.70)$

$$
\begin{aligned}
& e^{-\varepsilon^{-1} \operatorname{Im} \Delta_{12}\left(z_{1}\right)}\left|t_{21}(2,0)\right|=O(1), \\
& e^{-\varepsilon^{-1} \operatorname{Im} \Delta_{12}\left(z_{1}\right)}\left|t_{21}(6,4)\right|=O(1),
\end{aligned}
$$

which finally yield

$$
t_{21}(2,0)=e^{-i \theta_{12}^{*}} e^{-i \varepsilon^{-1} \int_{\eta} e_{1}^{*}}\left(1+O\left(e^{-\kappa \varepsilon^{-1}}\right)\right)
$$

This lemma and Corollary 3.4 allow us to obtain an asymptotic expression for $\ln S_{21}$ beyond all orders by integrating (3.18) from $-\infty$ to $+\infty$ along the paths described above. Let us recall that we have

$$
\operatorname{Im} \Delta_{12}\left(z_{1}\right) \equiv \operatorname{Im} \Delta_{12}\left(z_{i}\right), \quad i=1, \ldots, p .
$$


Thus, along the Stokes.lines we use the matrices given by Corollary 3.4 and which we can write as

(3.73) $\quad T:=T\left(z, z_{0}\right)=\left(\begin{array}{cc}1+O\left(e^{-\kappa \varepsilon^{-1}}\right) & O\left(e^{-\kappa \varepsilon^{-1}}\right) e^{-\varepsilon^{-1} \operatorname{Im} \Delta_{12}\left(z_{1}\right)} \\ O\left(e^{-\kappa \varepsilon^{-1}}\right) e^{\varepsilon^{-1} \operatorname{Im} \Delta_{12}\left(z_{1}\right)} & 1+O\left(e^{-\kappa \varepsilon^{-1}}\right)\end{array}\right)$

On the other hand, when we go from one Stokes line, $l_{j-1}$, to the next one, $l_{j}$, we use the matrix given by Lemma 3.6 :

(3.74) $\quad S_{j}:=\left(\begin{array}{cc}1+O\left(e^{-\kappa \varepsilon^{-1}}\right) & O\left(e^{-\kappa \varepsilon^{-1}}\right) e^{-\varepsilon^{-1} \operatorname{Im} \Delta_{12}\left(z_{1}\right)} \\ e^{-i / \varepsilon \int_{\eta_{j}} e_{i}^{*}} e^{-i \theta_{12}^{\star}(j)}\left(1+O\left(e^{-\kappa \varepsilon^{-1}}\right)\right) & 1+O\left(e^{-\kappa \varepsilon^{-1}}\right)\end{array}\right)$,

where $\int_{\eta_{j}} e_{1}^{\star}$ and $\theta_{21}^{\star}(j)$ are the quantities associated with the simple zero $z_{j}$ of $\rho(z)$ Therefore if we start at $-\infty$ with the values $c_{1}^{\star}(-\infty)=1$ and $c_{2}^{\star}(0)=0$, then the coefficients $c_{1}^{\star}(+\infty)$ and $c_{2}^{\star}(+\infty)$ are obtained by computing

$$
\left(\begin{array}{c}
c_{1}^{\star}(\infty) \\
c_{2}^{\star}(\infty)
\end{array}\right)=T S_{p} T S_{p-1} \ldots S_{1} T\left(\begin{array}{l}
1 \\
0
\end{array}\right)
$$

which proves the final theorem of this section, (restoring the $\varepsilon$ dependence):

THEOREM 3.7. Under Conditions I-III, the solution of (3.18) such that $c_{1}^{\star}(-\infty)=1$ and $c_{2}^{\star}(-\infty)=0$ is given at $x=+\infty$ by

$$
c_{1}^{\star}(\infty)=1+O\left(e^{-\kappa \varepsilon^{-1}}\right)
$$

and

$$
c_{2}^{\star}(\infty)=\sum_{k=1}^{p} e^{-i / \varepsilon \int_{\eta_{k}} e_{1}^{*}(z, \varepsilon) d z} e^{-i \theta_{12}^{*}(k, \varepsilon)}+O\left(e^{-\kappa \varepsilon^{-1}}\right) e^{\varepsilon^{-1} \operatorname{Im} \Delta_{12}\left(z_{1}\right)}
$$

where $\operatorname{Im} \int_{\eta_{k}} e_{1}^{\star}(z, \varepsilon) d z=\operatorname{Im} \Delta_{12}\left(z_{1}\right)+O\left(\varepsilon^{2}\right)$ and $\theta_{12}^{\star}(k, \varepsilon)=O(1)$.

4. Applications.

4.1. Explicit formulae. Let us start by deriving explicit formulae for the eigenvectors $\varphi_{j}^{\star}(z)$ of $A_{\mathrm{q}^{\star}}(z)$ defined by (3.10). They will then allow us to give the precise relation between the coefficients $c_{j}(z)$ defined by the expansion

$$
\varphi(z)=\sum_{j=1}^{2} c_{j}(z) e^{-i / \varepsilon \int_{0}^{z} e_{j}\left(z^{\prime}\right) d z^{\prime}} \varphi_{j}(z)
$$

and the coefficients $c_{j}^{\star}(z)$ defined by

$$
\varphi(z)=\sum_{j=1}^{2} c_{j}^{\star}(z) e^{-i / \varepsilon \int_{0}^{z} e_{j}^{\star}\left(z^{\prime}\right) d z^{\prime}} \varphi_{j}^{\star}(z) .
$$

Note that here we have chosen $z_{0}=0$. Consider the operator $A_{q^{*}}(z), z \in \Sigma$, where $\Sigma$ is a simply connected domain of $\tilde{\Omega}$. We can write

$$
A_{q^{\star}}(z)=\left(\begin{array}{cc}
i c^{\star}(z) & a^{\star}(z) \\
b^{\star}(z) & -i c^{\star}(z)
\end{array}\right)
$$

with

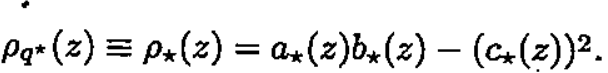

LEMMA 4.1. The eigenvectors of $A_{q^{*}}(z)$ defined by (3.10) are given by

$$
\varphi_{j}^{\star}(z)=\frac{\chi_{j}^{\star}(z)}{\left\|\chi_{j}^{\star}(-\infty)\right\|} e^{-i(-1)^{j} \sigma_{\star}(z)}, \quad j=1,2,
$$

where

$$
\chi_{j}^{\star}(z)=\left(\begin{array}{c}
\sqrt{\frac{a_{*}(z)}{\sqrt{\rho_{*}(z)}}} \\
(-1)^{j} \sqrt{\frac{\sqrt{\rho_{*}(z)}}{a_{*}(z)}}-i \frac{c_{*}(z)}{\sqrt{\sqrt{\rho_{*}(z) a_{*}(z)}}}
\end{array}\right)
$$

and

$$
\sigma_{\star}(z)=\frac{1}{2} \int_{-\infty}^{z} \frac{c_{\star}(u) a_{\star}^{\prime}(u)-c_{\star}^{\prime}(u) a_{\star}(u)}{\sqrt{\rho_{\star}(u)} a_{\star}(u)} d u
$$

for any. $z \in \Sigma \backslash Y_{\star}$ and $Y_{\star}=\left\{z \in \Sigma: a_{\star}(z)=0\right\}$.

Remarks. i) Any traceless matrix can be written under the form given above; the lemma actually requires the existence of distinct eigenvalues only. It is true in particular for the operator $A(z)$ written as in (4.3) without indices *.

ii) The vectors $\varphi_{j}^{\star}(z)$ are actually analytic in the whole set $\Sigma$ since the operator $W_{\star}(z)$ is analytic in $\Sigma$.

Proof. A direct verification shows that the vectors $\chi_{j}^{\star}(z)$ are eigenvectors of $A_{q^{\star}}(z)$ for the eigenvalues $e_{j}^{\star}(z)=(-1)^{j} \sqrt{\rho_{\star}(z)}$. We set the notation

$$
p_{\star}(z)=\sqrt{\rho_{\star}(z)}
$$

and we introduce the eigenprojectors (see (3.2))

$$
P_{q^{\star}, j}(z) \equiv P_{j}^{\star}(z)=\frac{1}{2}\left(\begin{array}{cc}
1+(-1)^{j} \frac{i c_{\star}(z)}{p_{\star}(z)} & (-1)^{j} \frac{a_{\star}(z)}{p_{\star}(z)} \\
(-1)^{j} \frac{b_{\star}(z)}{p_{\star}(z)} & 1-(-1)^{j} \frac{i c_{\star}(z)}{p_{\star}(z)}
\end{array}\right) .
$$

The vectors $\varphi_{j}^{\star}(z)$ must satisfy $P_{j}^{\star}(z) \varphi_{j}^{\star \prime}(z) \equiv 0$ (see (2.22)). We compute, dropping the arguments,

$$
\chi_{j}^{\star \prime}=\left(\begin{array}{c}
\frac{1}{2} \sqrt{\frac{p_{\star}}{a_{\star}}}\left(\frac{a_{\star}}{p_{\star}}\right)^{\prime} \\
\frac{(-1)^{j}}{2} \sqrt{\frac{a_{\star}}{p_{\star}}}\left(\frac{p_{\star}}{a_{\star}}\right)^{\prime}-i \frac{c_{\star}^{\prime}}{\sqrt{p_{\star} a_{\star}}}+\frac{i}{2} \frac{c_{\star}\left(p_{\star} a_{\star}\right)^{\prime}}{\left(p_{\star} a_{\star}\right)^{3 / 2}}
\end{array}\right)
$$

$$
P_{j}^{\star} \chi_{j}^{\star \prime}=i \frac{(-1)^{j}}{2} \frac{c_{\star} a_{\star}^{\prime}-c_{\star}^{\prime} a_{\star}}{p_{\star} a_{\star}} \chi_{j}^{\star} .
$$

Consequently, the vectors

$$
\varphi_{j}^{\star}=\frac{e^{-i\left((-1)^{j} / 2\right) \int_{-\infty}^{z}\left(c_{\star} a_{\star}^{\prime}-c_{\star}^{\prime} a_{\star} / p_{\star} a_{*}\right) d z}}{\left\|\chi_{j}^{\star}(-\infty)\right\|} \chi_{j}^{\star}
$$


normalized to 1 at $z=-\infty$, satisfy condition (2.22).

口

COROLLARY 4.2. Let $z_{k} \in X$ and let $\eta_{k}$ be a counterclockwise-oriented loop based at the origin which encircles the disc $D\left(z_{k}, r\right)$ only and passes through no point of $Y_{\star}$. Then the quantity $e^{i \theta_{12}^{*}(k)}$ defined in Lemma 3.1 is given by

$$
e^{i \theta_{12}^{\star}(k)}=-i e^{i \pi n_{k}^{\star}} \frac{\left\|\chi_{1}^{\star}(-\infty)\right\|}{\left\|\chi_{2}^{\star}(-\infty)\right\|} e^{-i / 2 \int_{\eta_{k}}\left(c_{*} a_{\star}^{\prime}-c_{\star}^{\prime} a_{\star} / \sqrt{p_{\star} a_{*}}\right) d z} e^{-2 i \sigma_{*}(0)}
$$

where $n_{k}^{\star} \in \mathrm{Z}$ depends on $a_{\star}$ and $\eta_{k}$.

Proof. It is always possible to choose a loop $\eta_{k}$ as described. By Lemma 3.1 we have

$$
\sqrt{\rho_{\star}\left(0 \mid \eta_{k}\right)}=e^{i \pi} \sqrt{\rho_{\star}(0)}
$$

and

$$
a_{\star}\left(0 \mid \eta_{k}\right)=e^{i 2 \pi n_{k}^{*}} a_{\star}(0)
$$

with $n_{k}^{\star} \in \mathbf{Z}$ since $a_{\star}(z)$ is single valued in $\tilde{\Omega}$. As a consequence

$$
\chi_{2}^{\star}\left(0 \mid \eta_{k}\right)=-i e^{i \pi n_{k}^{\star}} \chi_{1}^{\star}(0)
$$

Finally,

$$
\sigma_{\star}\left(0 \mid \eta_{k}\right)=\frac{1}{2} \int_{\eta_{k}} \frac{c_{\star} a_{\star}^{\prime}-c_{\star}^{\prime} a_{\star}}{\sqrt{\rho_{\star}} a_{\star}} d z+\sigma_{\star}(0)
$$

so that

(4.14)

$$
\varphi_{2}^{\star}\left(0 \mid \eta_{k}\right)=\varphi_{1}^{\star}(0)(-i) e^{i \pi n_{k}^{\star}} \frac{\left\|\chi_{1}^{\star}(-\infty)\right\|}{\left\|\chi_{2}^{\star}(-\infty)\right\|} e^{-i / 2 \int_{\eta_{k}}\left(c_{\star} c_{\star}^{\prime}-c_{\star}^{\prime} a_{\star} / \sqrt{p_{\star}} a_{\star}\right) d z} e^{-2 i \sigma_{\star}(0)}
$$

Consider now the two decompositions (4.1) and (4.2). The relation between the coefficients associated with the choice of eigenvectors made in Lemma 4.1 is given by the following corollary.

COROLLARY 4.3. The coefficients $c_{j}^{\star}( \pm \infty)$ and $c_{j}( \pm \infty)$ defined by (4.1), (4.2), and Lemma 4.1 are such that

$$
\begin{aligned}
& c_{j}(-\infty)=c_{j}^{\star}(-\infty) e^{-i / \varepsilon \int_{0}^{-\infty} e_{j}^{\star}(x)-e_{j}(x) d x} \\
& c_{j}(+\infty)=c_{j}^{\star}(+\infty) e^{-i(-1)^{j}\left(\sigma_{*}(+\infty)-\sigma(+\infty)\right)} e^{-i / \varepsilon \int_{0}^{+\infty} e_{j}^{\star}(x)-e_{j}(x) d x}
\end{aligned}
$$

for $j=1,2$.

Proof. We write the operator $A$ under the form

$$
A(z)=\left(\begin{array}{cc}
i c(z) & a(z) \\
b(z) & -i c(z)
\end{array}\right)
$$

where we can assume, without loss of generality, that

$$
\lim _{x \rightarrow \pm \infty} a(x)=a( \pm \infty) \neq 0
$$

Indeed, we can always perform a change of orthonormal basis which amounts to replacing $A(z)$ by $S^{-1} A(z) S$, where $S$ is a constant unitary matrix. Since the gap condition holds at $\pm \infty, A( \pm \infty) \neq 0$. Thus, we can bring nonzero elements in the upper right corner of the matrices $S^{-1} A( \pm \infty) S$ by taking for $S$ a rotation matrix in the plane of suitable angle. The corresponding eigenvectors $\varphi_{j}(z)$ are given by the expressions of Lemma 4.1 , where the indices $\star$ are dropped. Because the operators $A(x)$ and $A_{q^{\star}}(x)$ coincide at $|x|=\infty$, we have

$$
\chi_{j}^{\star}( \pm \infty)=\chi_{j}( \pm \infty)
$$

and

$$
\varphi_{j}^{\star}(-\infty)=\varphi_{j}(-\infty)
$$

Hènce

(4.19) $\quad \varphi_{j}^{\star}(+\infty)=\varphi_{j}(+\infty) e^{-i(-1)^{j}\left(\sigma_{\star}(+\infty)-\sigma(+\infty)\right)} \equiv e^{-i \beta_{j}^{*}} \varphi_{j}(+\infty)$,

so that formulae (2.43) and (2.45) apply. $\quad \square$

4.2. Invariants. Let us consider now the following three classes of operators $A(x)$ :

$$
A(x)=A(x)^{\dagger}, \quad x \in \mathbf{R},
$$

where $\dagger$ denotes the adjoint.

2)

$$
A(x)=\left(\begin{array}{cc}
i c(x) & a(x) \\
b(x) & -i c(x)
\end{array}\right), \quad a(x), b(x), c(x) \in \mathbf{R}, \quad x \in \mathbf{R} .
$$

$$
A(x)=i\left(\begin{array}{cc}
c(x) & \alpha(x) \\
\beta(x) & -c(x)
\end{array}\right), \quad \alpha(x), \beta(x), c(x) \in \mathbf{R}, \quad x \in \mathbf{R} .
$$

Note in particular that the operator $H(x)$ in equation (1.7) belongs to the first class whereas the operators in equations (1.9) and (1.11) belong to the second class. For these classes of operators there exist expressions involving the coefficients $c_{j}(x)$ and $c_{j}^{\star}(x)$ which are constant for all $x \in \mathbf{R}$.

LEMMA 4.4. If $A(x)$ belongs to class 1,2 , or 3 , then the operators $A_{q}(x)$ constructed by means of the iterative scheme (2.11), (2.12) belong to the same class, for any $q \leq q^{\star}$.

The proof of this lemma is obtained by a straightforward induction and will therefore be omitted.

LEMMA 4.5. i) If $A(x)$ belongs to class 1 , then

$$
\left|c_{1}(x)\right|^{2}+\left|c_{2}(x)\right|^{2}=\left|c_{1}^{\star}(x)\right|^{2}+\left|c_{2}^{\star}(x)\right|^{2} \equiv I, \quad x \in \mathbf{R}
$$

where I is constant. 
ii) If $A(x)$ belongs to class 2 or 3 , then

$$
\left|c_{1}(x)\right|^{2}-\left|c_{2}(x)\right|^{2}=\left|c_{1}^{\star}(x)\right|^{2}-\left|c_{2}^{\star}(x)\right|^{2} \equiv I, \quad x \in \mathbf{R},
$$

where $I$ is constant.

Proof. The first assertion is a direct consequence of the fact that $U\left(x, x_{0}\right)$, $W\left(x, x_{0}\right)$, and $W_{q^{\star}}\left(x, x_{0}\right)$ are unitary if $A(x)$ and $A_{q^{\star}}(x)$ are self-adjoint. Assume now that $A(x)$ belongs, to the second class and let

$$
G=\left(\begin{array}{cc}
1 & 0 \\
0 & -1
\end{array}\right)
$$

If $\varphi(x)$ is solution of equation (1.12),

$$
i \varepsilon \varphi(x)^{\prime}=A(x) \varphi(x),
$$

then $\overline{G \varphi(x)}$ is another solution of this equation. Indeed, $G^{2}=1$ so that we can write

$$
i \varepsilon \overline{G \varphi(x)}{ }^{\prime}=\overline{-G i \varepsilon \varphi(x)^{\prime}}=\overline{-G A(x) G G \varphi(x)}
$$

and we compute

$$
\overline{-G A(x) G}=A(x), \quad x \in \mathbf{R} .
$$

Therefore, as $\operatorname{tr} A(x) \equiv 0$, the following determinant is constant for any real $x$ :

$$
\operatorname{det}(\varphi(x), \overline{G \varphi(x)})=\text { constant. }
$$

Observe that the eigenvectors constructed in Lemma 4.1 satisfy the identity

$$
\overline{G \varphi_{j}(x)}=\varphi_{k}(x), \quad j \neq k
$$

since $\sigma(x)$ is real and $\left\|\chi_{j}(x)\right\|$ is independent of $j=1,2$ for real $a(x), b(x)$, and $c(x)$. Then we obtain from the reality of $e_{j}(x)$ and $e_{1}(x)=-e_{2}(x)$ that

(4.29) $\overline{G \varphi(x)}=\overline{c_{1}(x)} e^{-i / \varepsilon \int_{0}^{x} e_{2}\left(x^{\prime}\right) d x^{\prime}} \varphi_{2}(x)+\overline{c_{2}(x)} e^{-i / \varepsilon \int_{0}^{x} e_{1}\left(x^{\prime}\right) d x^{\prime}} \varphi_{1}(x)$.

It remains to use the multilinearity of the determinant to get

(4.30) $\quad \operatorname{det}(\varphi(x), \overline{G \varphi(x)})=\left(\left|c_{1}(x)\right|^{2}-\left|c_{2}(x)\right|^{2}\right) \operatorname{det}\left(\varphi_{1}(x), \varphi_{2}(x)\right)$;

we compute

$$
\operatorname{det}\left(\varphi_{1}(x), \varphi_{2}(x)\right)=2 \frac{\sqrt{\rho(-\infty)}}{a(-\infty)+b(-\infty)}
$$

using $\rho(x)=a(x) b(x)-(c(x))^{2}$. The identities (4.28) and (4.29) are also true for the eigenvectors $\varphi_{j}^{\star}(x)$ due to Lemma 4.4. Hence the same argument and (4.17) show that

(4.32) $\operatorname{det}(\varphi(x), \overline{G \varphi(x)})=\left(\left|c_{1}^{\star}(x)\right|^{2}-\left|c_{2}^{\star}(x)\right|^{2}\right) 2 \frac{\sqrt{\rho(-\infty)}}{a(-\infty)+b(-\infty)}=$ constant.
If $A(x)$ belongs to the third class, we proceed in a similar way. In this case, if $\varphi(x)$ is a solution of (1.12), $\overline{\varphi(x)}$ is another solution and we obtain from the explicit formulae of Lemma 4.1 (with the choice $\sqrt{i}=e^{i \pi / 4}$ )

$$
\overline{\varphi_{j}(x)}=-i \varphi_{k}(x) \text {. }
$$

Finally we compute

$$
\begin{aligned}
\operatorname{det}\left(\varphi(x), \frac{1}{\varphi(x)}\right) & =\left(\left|c_{1}(x)\right|^{2}-\left|c_{2}(x)\right|^{2}\right) 2 \frac{\sqrt{\rho(-\infty)}}{\beta(-\infty)-\alpha(-\infty)} \\
& =\left(\left|c_{1}^{\star}(x)\right|^{2}-\left|c_{2}^{\star}(x)\right|^{2}\right) 2 \frac{\sqrt{\rho(-\infty)}}{\beta(-\infty)-\alpha(-\infty)}=\text { constant. }
\end{aligned}
$$

Remark. It follows from (4.29) that if $\left(c_{1}(x), c_{2}(x)\right)$ are solutions of (3.18), then $\left(c_{2}(x), c_{1}(x)\right)$ provide another solution of $(3.18)$ when $A(x)$ belongs to class 2 or 3 . The corresponding symmetry property when $A(x)$ belongs to class 1 is that if $\left(c_{1}(x), c_{2}(x)\right)$ satisfy (3.18), then $\left(\overline{c_{2}(x)},-\overline{c_{1}(x)}\right)$ satisfy $(3.18)$ as well. This property can be derived from (3.18) directly by using the antiself-adjointness of $K_{q}(x), q \leq q^{\star}$ in this case [13].

4.3. Main applications. a) Let $A(x)$ be a $2 \times 2$ hermitian matrix, $x \in \mathbf{R}$, as in equation (1.7). The equation

$$
i \varepsilon \frac{d \varphi(x)}{d x}=A(x) \varphi(x), \quad \varepsilon \rightarrow 0
$$

describes the adiabatic limit of the dynamics of a two-level quantum mechanical system. The squared modulus of the element $S_{21}$ gives the probability $\mathcal{P}(\varepsilon)$ of a quantum transition over infinite time between the two eigenstates of the system.

COROLlary 4.6. If $A(x)$ is hermitian and satisfies Conditions I-III

$$
\mathcal{P}(\varepsilon)=\left|S_{21}\right|^{2}=\left|\sum_{k=1}^{p} e^{-i / \varepsilon \int_{\eta_{k}} e_{1}^{\star}(z, \varepsilon) d z} e^{-i \theta_{12}^{*}(k, \varepsilon)}\right|^{2}+O\left(e^{-\kappa \varepsilon^{-1}}\right) e^{\varepsilon^{-1} 2 \operatorname{Im} \Delta_{12}\left(z_{1}\right)} .
$$

b) Let $A(x)$ be the matrix (1.11)

$$
A(x)=\left(\begin{array}{cc}
0 & 1 \\
E-V(x) & 0
\end{array}\right)
$$

associated with the semiclassical regime of Schrödinger equation

$$
-\varepsilon^{2} \frac{d^{2} \psi(x)}{d x^{2}}+V(x) \psi(x)=E \psi(x), \quad \varepsilon \rightarrow 0,
$$

where $\inf _{x \in \mathbf{R}} E-V(x)>0$. A solution $\varphi(x)$ of (1.11) characterized by the asymptotic conditions $c_{1}(-\infty)=0, c_{2}(-\infty)=1$ describes a particle coming from the right whose energy is strictly above the potential barrier $V(x)$. The reflection coefficient $\mathcal{R}(\varepsilon)$ for this scattering process is then defined by $\mathcal{R}(\varepsilon)=\left|\frac{\mathcal{c}_{1}(+\infty)}{c_{c}(+\infty)}\right|^{2}$. As it stands here, it cannot be computed from the knowledge of $S_{21}$. However, as a consequence of Lemma 4.5 and the remark following it, we can write

$$
\mathcal{R}(\varepsilon)=\frac{\left|\tilde{c}_{2}(+\infty)\right|^{2}}{1+\left|\tilde{c}_{2}(+\infty)\right|^{2}}
$$


where $\tilde{c}_{1}(-\infty)=1$ and $\tilde{c}_{2}(-\infty)=0$. Hence we have the following corollary.

COROLLARY 4.7. If $A(x)$ given by (4.46) satisfies conditions I-III,

$\mathcal{R}(\varepsilon)=\frac{\left|S_{21}\right|^{2}}{1+\left|S_{21}\right|^{2}}=\left|\sum_{k=1}^{p} e^{-i / \varepsilon \int_{\eta_{k}} e_{1}^{*}(z, \varepsilon) d z} e^{-i \theta_{12}^{*}(k, \varepsilon)}\right|^{2}+O\left(e^{-\kappa \varepsilon^{-1}}\right) e^{\varepsilon^{-1} 2 \operatorname{Im} \Delta_{12}\left(z_{1}\right)}$.

c) Let $A(x)$ be the matrix

$$
A(x)=\left(\begin{array}{cc}
0 & 1 \\
\omega^{2}(x) & 0
\end{array}\right)
$$

associated with the equation of motion (1.9) of a classical oscillator whose frequency varies slowly with time

(4.40) $\quad \varepsilon^{2} \frac{d^{2} u(x)}{d x^{2}}=-\omega^{2}(x) u(x), \quad u(0)=u_{0}, \varepsilon \frac{d u(0)}{d x}=u_{1}, \quad \varepsilon \rightarrow 0$.

We assume that the initial values $u_{0}$ and $u_{1}$ are independent of $\varepsilon$. In terms of the variable $u(x)$, the adiabatic invariant (1.6) reads (keeping the same notation $J$ )

$$
J(x, \varepsilon)=\frac{\varepsilon^{2}\left|u^{\prime}(x)\right|^{2}+\omega^{2}(x)|u(x)|^{2}}{\omega(x)}
$$

Note that we do not require the initial values $u_{0}$ and $u_{1}$ to be real. Let us express $\Delta J(\varepsilon)$ in terms of the elements of the matrix $S$. We set

$$
\Omega(x)=\left(\begin{array}{cc}
\omega(x) & 0 \\
0 & \frac{1}{\omega(x)}
\end{array}\right)
$$

so that we have with $\varphi(x)$ defined by (1.8)

$$
J(x, \varepsilon)=\langle\varphi(x) \mid \Omega(x) \varphi(x)\rangle .
$$

Writing

$$
\varphi(x)=\sum_{j=1}^{2} d_{j}(x) e^{-i / \varepsilon \int_{0}^{x}(-1)^{j} \omega\left(x^{\prime}\right) d x^{\prime}} \varphi_{j}(x)
$$

where

$$
\varphi_{j}(x)=\left(\begin{array}{c}
\frac{1}{\sqrt{\omega(x)}} \\
(-1)^{j} \sqrt{\omega(x)}
\end{array}\right) \sqrt{\frac{\omega(-\infty)}{1+\omega^{2}(-\infty)}}
$$

we compute

$$
J(x, \varepsilon)=2 \frac{\omega(-\infty)}{1+\omega^{2}(-\infty)}\left(\left|d_{1}(x)\right|^{2}+\left|d_{2}(x)\right|^{2}\right)
$$

Let us introduce the coefficients $d_{j}^{\star}(x)$ by

$$
\varphi(x)=\sum_{j=1}^{2} d_{j}^{\star}(x) e^{-i / \varepsilon \int_{0}^{x} e_{j}^{\star}\left(x^{\prime}\right) d x^{\prime}} \varphi_{j}^{\star}(x)
$$

satisfying the initial condition

$$
\varphi(0)=\left(\begin{array}{c}
u_{0} \\
i u_{1}
\end{array}\right)=d_{1}^{\star}(0) \varphi_{1}^{\star}(0)+d_{2}^{\star}(0) \varphi_{2}^{\star}(0) .
$$

This last equation and Lemma 4.1 allow us to express the $d_{j}^{\star}(0)$ as functions of $u_{0}$ and $u_{1}$ and we have in particular $d_{j}^{\star}(0)=O(1)$. As a consequence of Corollary 4.3 we have $\left|d_{j}( \pm \infty)\right|=\left|d_{j}^{\star}( \pm \infty)\right|, j=1,2$, so that

(4.49) $\Delta J(\varepsilon)=2 \frac{\omega(-\infty)}{1+\omega^{2}(-\infty)}\left(\left|d_{1}^{\star}(+\infty)\right|^{2}+\left|d_{2}^{\star}(+\infty)\right|^{2}-\left|d_{1}^{\star}(-\infty)\right|^{2}-\left|d_{2}^{\star}(-\infty)\right|^{2}\right)$

Then it results from the linearity of equation (3.18) and from the remark following the proof of Lemma 4.5 that we can write

$$
\left(\begin{array}{l}
d_{1}^{\star}(x) \\
d_{2}^{\star}(x)
\end{array}\right)=\alpha(\varepsilon)\left(\begin{array}{l}
c_{1}^{\star}(x) \\
c_{2}^{\star}(x)
\end{array}\right)+\beta(\varepsilon)\left(\overline{\frac{c_{2}^{\star}(x)}{c_{1}^{\star}(x)}}\right),
$$

where the $c_{j}^{\star}(x)$ satisfy $(3.18)$ as well with boundary conditions $c_{1}^{\star}(-\infty)=1, c_{2}^{\star}(-\infty)=$ 0 . These boundary conditions together with equation (2.46) allow us to express the constants $\alpha(\varepsilon)$ and $\beta(\varepsilon)$ as functions of the $d_{j}^{\star}(0)$ which are defined by the initial condition (4.48):

$$
\left(\begin{array}{c}
d_{1}^{\star}(-\infty) \\
d_{2}^{\star}(-\infty)
\end{array}\right)=\left(\begin{array}{c}
\alpha(\varepsilon) \\
\beta(\varepsilon)
\end{array}\right)=\left(\begin{array}{c}
d_{1}^{\star}(0)+O\left(e^{-\kappa \varepsilon^{-1}}\right) \\
d_{2}^{\star}(0)+O\left(e^{-\kappa \varepsilon^{-1}}\right)
\end{array}\right)
$$

We can now express the total variation of the adiabatic invariant as a function of the matrix $S$ and the initial conditions using (4.49) and Lemma 4.5:

$$
\begin{aligned}
\Delta J(\varepsilon)=2 \frac{\omega(-\infty)}{1+\omega^{2}(-\infty)}[ & {\left[4 \operatorname{Re}\left\{\alpha(\varepsilon) \overline{\beta(\varepsilon)} c_{1}^{\star}(+\infty) c_{2}^{\star}(+\infty)\right\}\right.} \\
& \left.+2\left|c_{2}^{\star}(+\infty)\right|^{2}\left(|\alpha(\varepsilon)|^{2}+|\beta(\varepsilon)|^{2}\right)\right]
\end{aligned}
$$

Hence, by (4.51) and Corollary 4.3, we have the following corollary. COROLlarY 4.8. If $A(x)$ given by (4.39) satisfies conditions I-III,

$$
\begin{aligned}
\Delta J(\varepsilon)= & 2 \frac{\omega(-\infty)}{1+\omega^{2}(-\infty)}\left[4 \operatorname{Re}\left\{d_{1}(-\infty) \overline{d_{2}(-\infty)} e^{+2 i / \varepsilon \int_{0}^{-\infty}\left(e_{1}^{*}(x, \varepsilon) \rightarrow e_{1}(x)\right) d x} S_{11} S_{21}\right\}\right. \\
& \left.+2\left|S_{21}\right|^{2}\left(\left|d_{1}(-\infty)\right|^{2}+\left|d_{2}(-\infty)\right|^{2}\right)\right] .
\end{aligned}
$$

If $d_{1}(-\infty) \overline{d_{2}(-\infty)}=0$

$$
\begin{aligned}
\Delta J(\varepsilon)= & 4 \frac{\omega(-\infty)}{1+\omega^{2}(-\infty)}\left|\sum_{k=1}^{p} e^{-i / \varepsilon \int_{\eta_{k}} e_{1}^{*}(z, \varepsilon) d z} e^{-i \theta_{12}^{*}(k, \varepsilon)}\right|^{2} \cdot\left(\left|d_{1}(-\infty)\right|^{2}+\left|d_{2}(-\infty)\right|^{2}\right) \\
& +O\left(e^{-\kappa \varepsilon^{-1}}\right) e^{\varepsilon^{-1} 2 \operatorname{Im} \Delta_{12}\left(z_{1}\right)}
\end{aligned}
$$




$$
\text { If } d_{1}(-\infty) \overline{d_{2}(-\infty)} \neq 0
$$

$$
\begin{aligned}
\Delta J(\varepsilon)= & 8 \frac{\omega(-\infty)}{1+\omega^{2}(-\infty)} \operatorname{Re}\left\{d_{1}^{\star}(0) \overline{d_{2}^{\star}(0)} \sum_{k=1}^{p} e^{-i / \varepsilon \int_{\eta_{k}} e_{1}^{\star}(z, \varepsilon) d z} e^{-i \theta_{12}^{*}(k, \varepsilon)}\right\} \\
& +O\left(e^{-\kappa \varepsilon^{-1}}\right) e^{\varepsilon^{-1} \operatorname{Im} \Delta_{12}\left(z_{1}\right)}
\end{aligned}
$$

where the quantities $d_{j}^{\star}(0)=O(1)$ are determined by the initial condition (4.48).

Remark. i) The coefficients $d_{j}$ are $O(1)$ since the initial conditions $u_{0}$ and $u_{1}$ are independent of $\varepsilon$.

ii) The condition $d_{1}(-\infty) \overline{d_{2}(-\infty)} \neq 0$ is equivalent to $d_{1}(0) \overline{d_{2}(0)} \neq 0$. From (4.45) and (4.48) we compute

$$
\begin{aligned}
& d_{1}(0)=\frac{1}{2} \sqrt{\frac{1+\omega^{2}(-\infty)}{\omega(-\infty)}}\left(u_{0} \sqrt{\omega(0)}-\frac{i}{\sqrt{\omega(0)}} u_{1}\right), \\
& d_{2}(0)=\frac{1}{2} \sqrt{\frac{1+\omega^{2}(-\infty)}{\omega(-\infty)}}\left(u_{0} \sqrt{\omega(0)}+\frac{i}{\sqrt{\omega(0)}} u_{1}\right),
\end{aligned}
$$

so that $d_{1}(-\infty) \overline{d_{2}(-\infty)} \neq 0$ is equivalent to $u_{1} \neq \pm i \omega(0) u_{0}$. This condition is always true for real initial values $u_{0}$ and $u_{1}$.

Appendix. We briefly describe in this appendix an explicit example of potential $V(x)$ for which the semiclassical above barrier reflection coefficient can be computed by applying the general theory developed in this paper. Consider the potential

$$
V(x)=\frac{1}{1+x^{4}}
$$

and choose an energy level $E>1$. Then the function

$$
p^{2}(x) \equiv \rho(x)=E-\frac{1}{1+x^{4}}
$$

is positive for any $x \in \mathbf{R}$. This function is meromorphic in $\mathbf{C}$ with first-order poles at the points

$$
\dot{y_{k}}=e^{i((\pi / 4)+k(\pi / 2))}, \quad k=0,1,2,3
$$

and first-order zeros at the points

$$
z_{k}=\left(1-\frac{1}{E}\right)^{1 / 4} e^{i((\pi / 4)+k(\pi / 2))}, \quad k=0,1,2,3
$$

Hence the matrix $A(x)$ given by (1.11) has an analytic continuation in the set $\Omega \equiv$ $\mathbf{C} \backslash\left\{y_{1}, y_{2}, y_{3}, y_{4}\right\}$. The Stokes lines are obtained by studying the level lines of the multivalued function $\int_{0}^{z} d z^{\prime} p\left(z^{\prime}\right)$ in the set $\Omega$. By a numerical study, we see that these lines behave in the first quadrant of the complex plane as described in Fig. 7.

We can show by exploiting the symmetries of the function $\rho$ that these lines are symmetric with respect to both the real and imaginary axes. Hence, Conditions I, II, and III are satisfied and the above barrier reflection coefficient can be computed

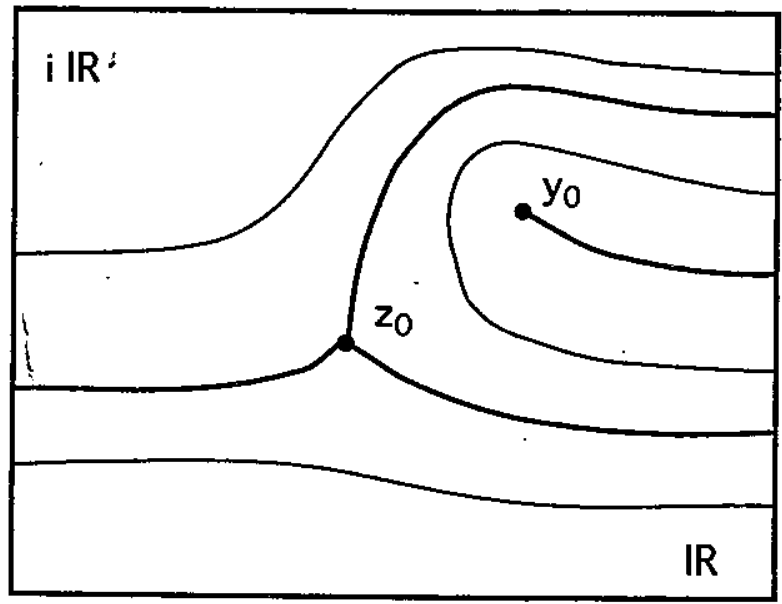

FIG. 7. The level lines of $\int_{0}^{z} d z^{\prime} p\left(z^{\prime}\right)$ in the first quadrant of the complex plane.

asymptotically as $\hbar$ goes to zero using the method explained above. In particular, we see from Corollary 4.2 that in the first-order asymptotic formula, $\theta_{12}(k), k=0,1$ is real since the function $c(z) \equiv 0$ and $\left\|\chi_{1}( \pm \infty)\right\|=\left\|\chi_{2}( \pm \infty)\right\| ;$ see (4.7). Hence it remains to compute $\int_{\eta_{k}} p(z) d z, k=1,2$, to get the first-order asymptotic formula for $\mathcal{R}(\hbar)$. Moreover, the presence of two first-order zeros in the upper half-plane linked by a Stokes line shows that an interference phenomenon takes place (Stückelberg oscillations) at the first order already, even though the potential barrier displays one bump only. The high-order corrections can be systematically computed using the theory developed in this paper; we omit this computational aspect here.

Acknowledgments. We thank the referee for constructive suggestions and C. Ballif for computing numerically the Stokes lines of the example in the appendix.

\section{REFERENCES}

[1] N. Fröman AND P. O. Fröman, JWKB Approximation, Contributions to the Theory, North Holland, Amsterdam, 1965

[2] W. WASow, Asymptotic Expansions for Ordinary Differential Equations, John Wiley Intersciences, New York, 1965.

[3] M. V. FEDORIUK, Méthodes Asymptotiques pour les Equations Différentielles Ordinaires Linéaires, Mir Moscou, 1987.

[4] Рн. A. Martin AND G. Nenciv, Semi-classical inelastic S-matrix for one-dimensional Nstate systems, Proc. Leuven Conference on the Three Levels, Micro-, Meso-, and MacroApproaches in Physics, M. Fannes, C. Meas, and A. Verbeure, eds., Plenum Press, New

York, 1994.

5] J. DAVIS AND P. PEChUKaS, Nonadiabatic transitions induced by a time-dependent Hamiltonian in the semiclassical/adiabatic limit: The two-state case, J. Chem. Phys., 64 (1976), pp.

6] J.-T. HWANG AND P. PECHUKAS, The adiabatic theorem in the complex plane and the semiclassical calculation of non-adiabatic transition amplitudes, J. Chem. Phys., 67 (1977), pp.

[7] A. JOYE, H. KUNZ, AND C.-E. PFISTER, Exponential decay and geometric aspect of transition probabilities in the adiabatic limit, Ann. Phys., 208 (1991), pp. 299-332.

[8] M. V. BerRY, Geometric Amplitude Factors in Adiabatic Quantum Transitions, Proc. Roy. Soc. London Ser. A, 430 (1990), pp. 405-411. 
[9] R. E. MEYER, Adiabatic variation, Part II. Action change for the simple oscillator, J. Appl. Math. Phys., 24 (1973), pp. 517-524.

10] W. WAsow, Calculation of an adiabatic invariant by turning point theory, SIAM J. Math. Anal., 5 (1974), pp. 673-700.

[11] M. V. FEDORIUK, An adiabatic invariant for a system of linear oscillators and scattering theory, Differential Equations, 12 (1976), pp. 713-718.

12] J. B. KELLER AND Y. MU, Changes in adiabatic invariants, Ann. Phys., 205 (1991), pp. 219

227.
[13] A. JOYE, G. MILETI, AND C.-E. PFISTER, Interferences in adiabatic transition probabilities mediated by Stokes lines, Phys. Rev. A, 44 (1991), pp. 4280-4295.

[14] N. FRÖMiAN, Connection formulas for certain higher order phase-integral approximations, Ann.

Phys., $61(1970)$, pp. $451-464$.
[15] B. LUNDBORG, Phase-integral treatment of wave reflection by real potentials, Math. Proc. Cambridge Philos. Soc., 85 (1979), pp. 493-522.

Probabilities in the adi abatic limit, J. Phys. A, 24 (1991), pp. 753-766.

[17] S. G. KREIN, Linear Differential Equations in Banach Spaces, American Mathematical Society, Providence, RI, 1971.

[18] T. Kato, Perturbation Theory for Linear Operators, Springer-Verlag, Berlin, Heidelberg, New

19] A. JOYE AND C.-E. PFISTER, Superadiabatic evolution and adiabatic transition probability between two non-degenerate levels isolated in the spectrum, J. Math. Phys., 34 (1993), pp. 454-479.

[20] G. NENCIU, Linear adiabatic theory: Exponential estimates, Comm. Math. Phys., 152 (1993), pp. $479-496$.

21] A. MARTINEZ, Precise exponential estimates in adiabatic theory, Université Paris-Nord, 1993, preprint.

[22] J. SJóstrand, Projecteurs adiabatiques du point de vue pseudodifférentiel, C.R. Acad. Sci. Paris Sér. I Math, 317 (1993), pp. 217-220.

[23] A. JOYE AND C.-E. PFISTER, Exponentially small adiabatic invariant for the Schrödinger equation, Comm. Math. Phys., 140 (1991), pp. 15-41.

[24] A. JOYE, Non-trivial prefactors in adiabatic transition probabilities induced by high order complex degeneracies, J. Phys. A, 26 (1993), pp. 6517-6540. 